\title{
Shapley Supercluster Survey: mapping the filamentary network connecting the clusters
}

\author{
C. P. Haines, ${ }^{1,2 \star}$ G. Busarello, ${ }^{3}$ P. Merluzzi, ${ }^{3 \star}$ K. A. Pimbblet, ${ }^{4}$ F. P. A. Vogt,${ }^{5} \dagger$ \\ M. A. Dopita, ${ }^{6}$ A. Mercurio, ${ }^{3}$ A. Grado, ${ }^{3}$ and L. Limatola ${ }^{3} \ddagger$ \\ ${ }^{1}$ INAF - Osservatorio Astronomico di Brera, via Brera 28, I-20121 Milano, Italy \\ ${ }^{2}$ Departamento de Astronomía, Universidad de Chile, Casilla 36-D, Correo Central, Santiago, Chile \\ ${ }^{3}$ INAF - Osservatorio Astronomico di Capodimonte, via Moiariello 16, I-80131 Napoli, Italy \\ ${ }^{4}$ E.A. Milne Centre for Astophysics, University of Hull, Cottingham Road, Kingston-upon-Hull, HU6 7RX, UK \\ ${ }^{5}$ European Southern Observatory, Av. Alonso de Córdova 3107, Vitacura, Casilla 19001, Santiago, Chile \\ ${ }^{6}$ Research School of Australia and Astrophysics, Australian National University, Canberra, ACT 2611, Australia
}

Accepted 2018 August 21. Received 2018 August 21; in original form 2018 July 2

\begin{abstract}
We have mapped the structure and connectivity of the Shapley supercluster core and its surroundings as traced by its member galaxies. To achieve this, we have carried out a new redshift survey of galaxies across a 21 square degree region $\left(17 \times 17 \mathrm{Mpc}^{2}\right)$ of the Shapley supercluster containing 11 rich clusters, all lying at $z \sim 0.048$. This survey aims to provide full spectroscopic coverage of galaxies for the Shapley Supercluster Survey (ShaSS), a multiwavelength survey based on high-quality optical ugri and near-infrared $K$-band imaging acquired with the 2.6-m VST and 4.1-m VISTA telescopes. Targets were selected from the VST images as having $i<18.0$ and WISE/W1 $<15.5 \mathrm{mag}$, and the observations were carried out with the AAOmega spectrograph on the 3.9-m Anglo-Australian Telescope. We present here redshift measurements for 4027 galaxies, of which 957 belong to the Shapley Supercluster. Including existing measurements, there are now reliable redshifts for 95 per cent of galaxies within the survey's magnitude limits over the $21 \mathrm{deg}^{2}$ region. This has enabled us to: (i) obtain a detailed map of the galaxy number density across the whole region; (ii) identify new groups and cluster substructures; (iii) establish the existence of a stream of galaxies connecting A 3559 to the supercluster core. We provide updated central redshifts and velocity dispersions of the 11 clusters, confirming that they all lie within $1300 \mathrm{~km} \mathrm{~s}^{-1}$ of the central cluster Abell 3558. These 11 systems are all inter-connected and lie within a coherent sheet of galaxies that fills the entire survey region without gaps. Clear velocity caustics extend right to the survey boundary, indicating that the entire structure is gravitationally bound and in the process of collapse.
\end{abstract}

Key words: catalogues, galaxies: clusters: general-large-scale structure of Universecosmology: observations-galaxies: clusters: individual: A3552, A3554, A3556, A3558, A3559, A3560, A3562.

\section{INTRODUCTION}

The structure of the Universe on the largest scales can be described as the cosmic web, a filamentary network connecting groups and clusters of galaxies (Joeveer \& Einasto 1978; Gott, Dickinson \& Melott 1986; Pimbblet, Drinkwater \& Hawkrigg 2004; Guzzo et al. 2014). The largest coherent structures within this cosmic web are

^E-mail: cphaines123@gmail.com (CPH); paola.merluzzi@inaf.it (PM) $\dagger$ ESO fellow.

$\ddagger$ AAOmega observations have been financed by the OPTICON consortium. the superclusters, forming out of initial density perturbations on scales of $100 \mathrm{Mpc}$ (Zeldovich, Einasto \& Shandarin 1982; Bond, Kofman \& Pogosyan 1996; Springel, Frenk \& White 2006), and containing dozens of galaxy clusters and groups (e.g. Einasto et al. $2011 \mathrm{a}, \mathrm{b})$ which are frequently interacting and merging. They mark the transition from the linear to the non-linear regime. While the full extent of superclusters may not be gravitationally bound, they contain high-density cores which have already collapsed to form clusters (Einasto et al. 2016), or larger overdensities which are currently in the process of collapsing to form what will become the most massive virialized structures in the distant future (Dünner et al. 2006; Araya-Melo et al. 2009; Pearson 2015). 
The most massive supercluster in the local Universe is the Shapley supercluster, first identified as a 'remote cloud of galaxies in Centaurus' by Shapley (1930). It encompasses at least 25 Abell clusters in the redshift range $0.035<z<0.06$ over a $\sim 15 \times 10 \mathrm{deg}^{2}$ region (Raychaudhury 1989; Ettori, Fabian \& White 1997; Einasto et al. 2001; de Filippis, Schindler \& Erben 2005; Proust et al. 2006a,b), distributed across the sky as shown in Fig. 1.

At the heart of the Shapley supercluster complex is the highdensity Shapley Supercluster Core (SSC) at $z=0.048$ comprising three Abell clusters (A 3558, A 3562, and A 3556) and two poor clusters (SC 1327-312 and SC 1329-313), forming a continuous filamentary structure 2 degrees $(\sim 8 \mathrm{Mpc})$ in extent, that is filled with hot gas as seen by both Planck and XMM-Newton satellites (Planck Collaboration et al. 2014; Merluzzi et al. 2016).

X-ray observations and redshift surveys focussed on the SSC have shown that it is perhaps the most complex and dynamically active region in the local Universe (Bardelli et al. 1998a), with multiple signs of ongoing and past cluster-cluster interactions and mergers (Bardelli et al. 1998a; Finoguenov et al. 2004; Rossetti et al. 2007), and galaxies suffering frequent high-velocity $\left(>2000 \mathrm{~km} \mathrm{~s}^{-1}\right)$ encounters with other galaxies and clusters (Reisenegger et al. 2000). This has motivated detailed surveys of this region to quantify the impact of these extreme environments on galaxy evolution, as revealed through the luminosity and stellar mass functions of supercluster galaxies (Mercurio et al. 2006; Merluzzi et al. 2010), the stellar populations and fundamental plane of early-type galaxies in the SSC (Smith, Lucey \& Hudson 2007; Gargiulo et al. 2009), and the star-formation activity of supercluster galaxies through bespoke Spitzer mid-infrared imaging of the SSC (Haines et al. 2011a,b,c, 2013). More recently, we have identified supercluster galaxies undergoing ram-pressure stripping as they encounter the ICM which pervades the SSC, and used integral-field spectroscopy to examine its effects upon the gas and stellar components of the galaxy in detail (Merluzzi et al. 2013, 2016).

The above studies all relied upon optical imaging (the Shapley Optical Survey; Haines et al. 2006; Mercurio et al. 2006) that covered a $\sim 2 \times 1 \mathrm{deg}^{2}$ field which barely enclosed the virialized regions of A 3556, A3358, and A 3562, meaning that environmental trends could only be followed out to $r_{200}$. However, it is known that the star formation activity of galaxies is already being affected by cluster-related mechanisms out at 3-5 $r_{200}$ (Bahé et al. 2013; Haines et al. 2015), while back-splash galaxies which have recently passed through the cluster cores are expected to rebound to $2 r_{200}$ (Mamon et al. 2004; Pimbblet 2011). Moreover, galaxies in the infall regions (1-4 $\left.r_{200}\right)$ of clusters have been observed to be transformed within filaments (Porter et al. 2008; Kleiner et al. 2017) or by preprocessing within groups (Mahajan, Haines \& Raychaudhury 2010; Jaffé et al. 2016; Bianconi et al. 2018; Haines et al. 2018), prior to their accretion into the clusters themselves. This all motivates surveys of cluster galaxies which extend well beyond $r_{200}$ to identify the sites where star-forming spirals on their first infall are first affected by their environment. There is further incentive to widen the analysis in the case of Shapley, with multiple Abell clusters in the immediate vicinity of the SSC that have redshifts within a few hundred $\mathrm{km} \mathrm{s}^{-1}$ of A 3558 (Reisenegger et al. 2000; Proust et al. 2006a), suggestive of being physically connected (Fig. 1) and gravitationally bound. Moreover, the Shapley Supercluster lies in the direction of the dipole anisotropy of the cosmic microwave background, and the relevance of its gravitational pull upon the high peculiar velocity $\left(\sim 600 \mathrm{~km} \mathrm{~s}^{-1}\right)$ of the Local Group relative to the Hubble Flow remains an open issue (Raychaudhury 1989; Kocevski, Mullis \& Ebeling 2004; Courtois et al. 2017).
With all the above in mind, we have undertaken the Shapley Supercluster Survey (ShaSS), a multiwavelength survey of a $23 \mathrm{deg}^{2}$ region $\left(17 \times 17 \mathrm{Mpc}^{2}\right)$ centred on the SSC. Its aim is to trace the filaments feeding the SSC, and reveal how the surrounding clusters are connected to each other and the Shapley supercluster core. As such, its extent (green box in Fig. 1) was designed to cover nine Abell clusters (A 3552, A 3554, A 3556, A 3558, A 3559, A 3560, A 3562, AS0724, AS0726) and two poor clusters (SC1327-312, SC1329-313) whose redshifts all lie within $1500 \mathrm{~km} \mathrm{~s}^{-1}$ of Abell 3558. ShaSS comprises high-quality optical ugri imaging carried out with OmegaCAM on the 2.6m VLT Survey Telescope (VST) and near-infrared $K$-band imaging from the $4.1 \mathrm{~m}$ Visible and Infrared Survey Telescope for Astronomy (VISTA), both taking advantage of the exceptional observing conditions available at Cerro Paranal in Chile. Full details of the VST and VISTA observations and overall scientific objectives of ShaSS are presented in Merluzzi et al. (2015). In this article, we present the spectroscopic component of ShaSS: a redshift survey of the same region carried out using the AAOmega fibre-fed multiobject spectrograph at the $3.9 \mathrm{~m}$ Anglo Australian Telescope in 2013 May.

The key goal of the AAOmega survey is to provide highly complete and homogeneous redshift coverage across the full ShaSS region. While there have been multiple previous redshift surveys of this region, these have either been much shallower (6dFGRS; Jones et al. 2009) or highly targetted towards individual clusters (e.g. Smith et al. 2004b, 2007; Moretti et al. 2017). This AAOmega survey effectively goes three magnitudes deeper than the 6dFGRS, filling in the undersampled filamentary regions that connect the clusters. This is vital for our aims to robustly map the large-scale structure surrounding A 3558 and connecting it to the neighbouring clusters. It is also fundamental to uniformly map the local environment of galaxies to reliably quantify the effects of environment on the galaxies within the Shapley supercluster.

The redshift survey enables the velocity limits of the Shapley supercluster to be robustly defined, and the confirmation of galaxies as supercluster members. This is a key step in the identification of promising targets for follow-up IFU spectroscopy through our ongoing WiFeS survey of ram-pressure stripped or interacting galaxies within the Shapley supercluster (Merluzzi et al. 2013, 2016, 2018).

We adopt a $\Lambda$ CDM cosmological model with $\Omega_{\mathrm{M}}=0.3, \Omega_{\Lambda}=$ 0.7 , and $H_{0}=70 \mathrm{~km} \mathrm{~s}^{-1}$. At the central redshift of the Shapley supercluster $(z=0.048)$, this gives an angular scale of $3.39 \mathrm{Mpc}$ degree $^{-1}$ and distance modulus of $36.66 \mathrm{mag}$.

\section{THE SAMPLE SELECTION BASED ON THE SHAPLEY SUPERCLUSTER SURVEY}

The ShaSS (Merluzzi et al. 2015) is based on VST optical imaging (Capaccioli \& Schipani 2011), acquired within the Italian INAF Guaranteed Time of Observations (PI: P. Merluzzi). The OmegaCAM instrument (Kuijken 2011) on the VST has a corrected field of view of $1^{\circ} \times 1^{\circ}$, enabling the whole ShaSS area to be covered with 23 VST fields as shown in Fig. 2. Each field was observed in four optical bands, $u\left(t_{\exp }=2955 \mathrm{~s}\right), g(1400 \mathrm{~s}), r(2664 \mathrm{~s})$, and $i$ $(1000 \mathrm{~s})$, reaching $5 \sigma(\mathrm{AB})$ magnitude limits of 24.4, 24.6, 24.1, and 23.3, respectively (Mercurio et al. 2015). While observations for all four bands took place contemporaneously from 2012 February onwards, the highest priority was given to the $i$-band observations, to ensure that complete coverage of the entire survey region was obtained in one band as soon as possible, in order to prepare tar- 


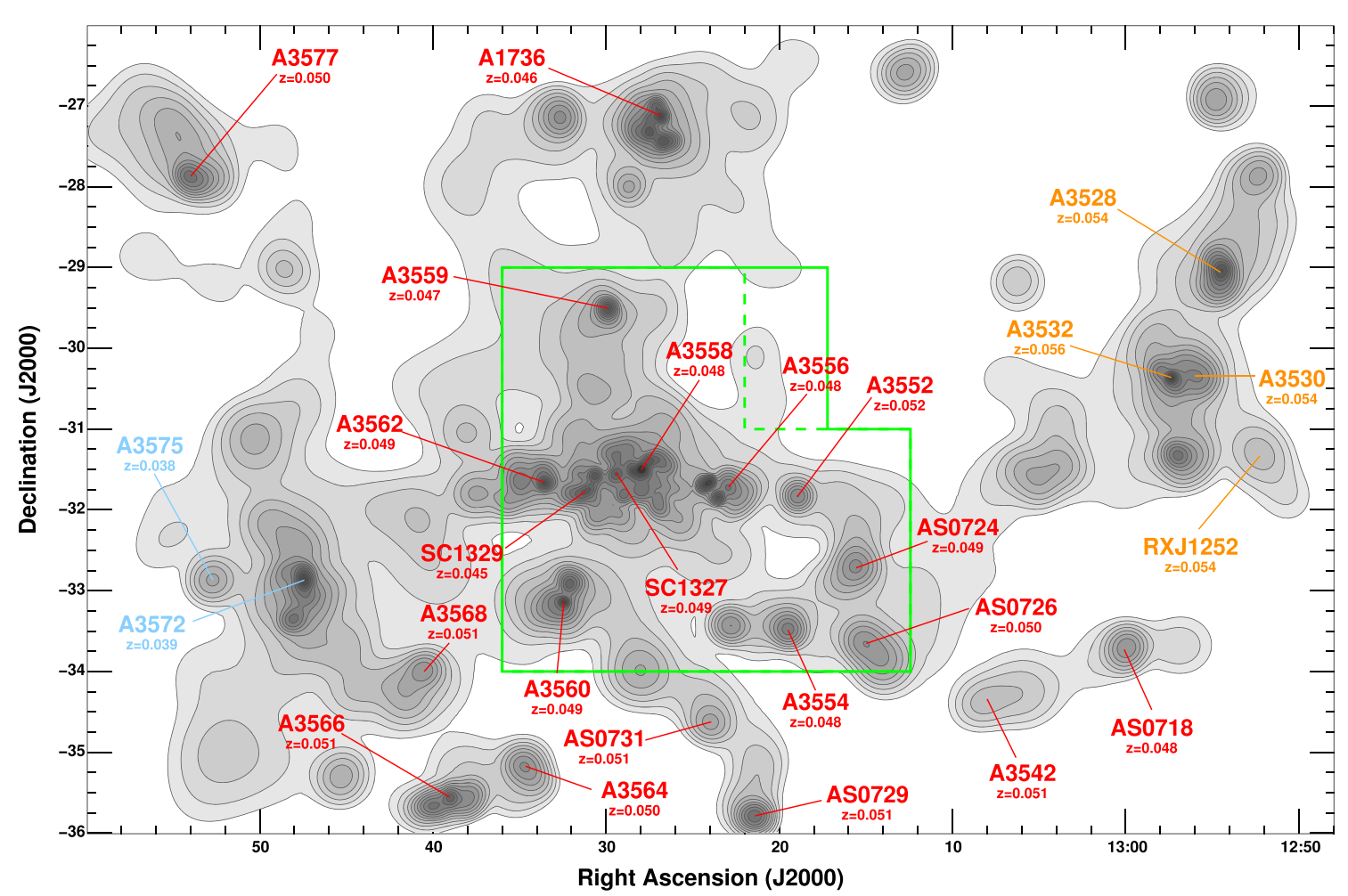

Figure 1. $K$-band luminosity-weighted density map of $0.035 \leq z<0.060$ galaxies across the full extent of the Shapley Supercluster. $K$-band magnitudes are taken from the 2MASS Extended Source Catalog and combines redshifts from the previous wide-field spectroscopic surveys covering the region (6dFGRS; Jones et al. 2009; Quintana et al. 1997; Kaldare et al. 2003; Drinkwater et al. 2004; Proust et al. 2006a; Cava et al. 2009). Abell clusters within the same redshift range are labelled, colour coded as: (red) recession velocities within $1500 \mathrm{~km} \mathrm{~s}^{-1}$ of the central cluster Abell 3558 ; (cyan) low-velocity extension $0.035 \leq z$ $<0.040$; (orange) high-velocity wing, $0.053 \leq z<0.060$. The solid green box outlines the $23 \mathrm{deg}^{2}$ region covered by ShaSS, and the dashed green lines our $21 \mathrm{deg}^{2}$ AAOmega survey.

gets for the redshift survey. ${ }^{1}$ The VST images have been processed and photometrically calibrated using the VST-Tube pipeline (Grado et al. 2012), and catalogues produced as described in Mercurio et al. (2015). The $i$-band images have median full width at half-maximum (FWHMs) of 0.65 arcsec, fully sampled by the 0.21 arcsec pixels.

At the time of AAOmega observations (2013 May), the $i$-band VST survey had completed 21 out of the 23 fields, with fields 19 and 20 (see Fig. 2) still to be observed. The AAOmega survey is thus limited to that area of 21 square degrees. The selection of targets for spectroscopic follow-up was made using the $i$-band photometric catalogue of Mercurio et al. (2015), considering only detections classified as galaxies with a total (Kron) magnitude brighter than $i$ $=18.0$ (AB mag, without correcting for Galactic extinction). The VST images enable robust star-galaxy separation to at least $i=$ 21.0 (Mercurio et al. 2015) and galaxy positions are accurate to $<0.1$ arcsec.

\subsection{WISE near-infrared photometry}

We exploit data from the Wide-field Infrared Survey Explorer (WISE; Wright et al. 2010) to provide infrared photometry for our galaxies in four infrared bands: 3.4, 4.6, 12, and $22 \mu \mathrm{m}$ ( $W 1$ to $W 4$, respectively). The WISE counterparts of galaxies in our $i$-band catalogue are identified by cross-correlating them with the WISE

${ }^{1}$ VST observations were carried out in service mode only, and observations in $i$-band had the least demanding scheduling constraints, enabling them to be completed earlier than the other three wavebands (ugr).
All-Sky Source Catalog ${ }^{2}$ within a matching radius of 3.5 arcsec. For most galaxies, the WISE magnitudes used are those measured with profile-fitting photometry (w1mpro), which are optimized for unresolved sources. For the brighter supercluster galaxies well resolved by the $\sim 6$ arcsec point spread function (PSF) of WISE (W1-W3), their magnitudes are measured inside elliptical apertures (w1gmag) matched to those from the associated 2MASS Extended Source Catalog (XSC; Jarrett et al. 2000) source. These elliptical apertures are found to correspond well to the Kron apertures used in our $i$-band magnitudes.

WISE imaged the entire sky with multiple exposures during its full cryogenic survey, and surveyed $\sim 20$ per cent of the sky a second time before its cryogen supply was exhausted. The Shapley supercluster lies within this 20 per cent, and so has around double the coverage depth of the main all-sky mission, giving $5 \sigma$ point source Vega magnitude limits of 17.5 (W1), 16.0 (W2), 11.8 (W3), and $8.2(W 4)$.

\subsection{Sample selection}

To obtain a roughly stellar mass $(\mathcal{M})$ limited galaxy sample, AAOmega fibres were placed on targets primarily based on their WISE $W 1(3.4 \mu \mathrm{m})$ magnitudes. The highest priority was given to galaxies with $W 1 \leq 13.0 \mathrm{mag}(\sim K *+1.2)$, which corresponds to a stellar mass limit of $2.7 \times 10^{10} \mathrm{M}_{\odot}$ (assuming

\footnotetext{
${ }^{2}$ http://wise2.ipac.caltech.edu/docs/release/allsky/expsup/
} 


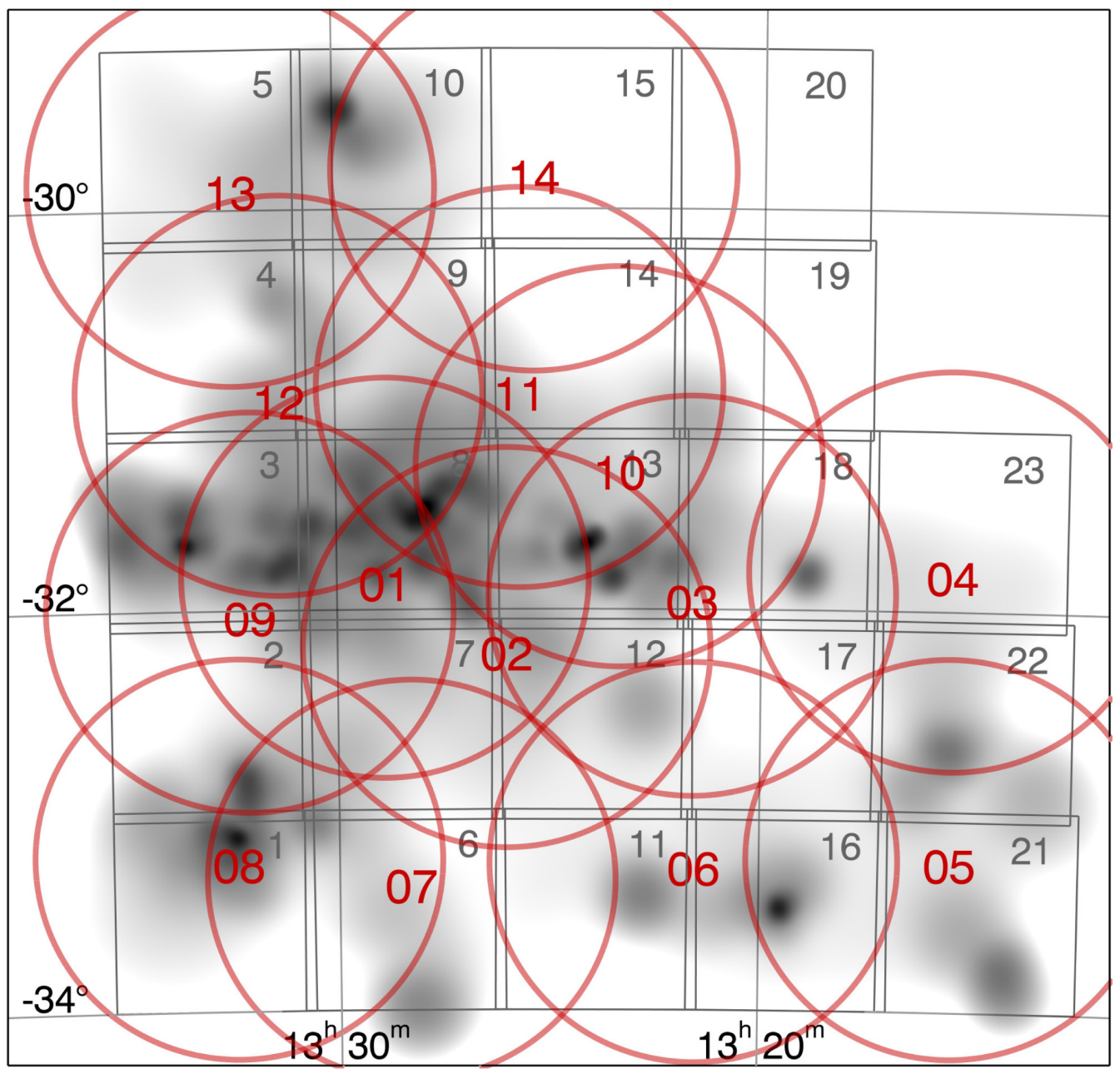

Figure 2. Coverage of the 14 AAOmega pointings (numbered red circles), compared to the 23 VST pointings with $i$-band imaging (grey squares) from the Shapley Supercluster Survey. The greyscale density map indicates the surface density of W1 flux emitted from galaxies identified as cluster members, including the new AAOmega redshifts, as derived in Merluzzi et al. (2015).

$\mathcal{M} / L_{W 1}=0.47 \mathrm{M}_{\odot} / \mathrm{L}_{\odot} ;$ McGaugh \& Schombert 2014). Having ensured maximal completeness for the massive $\gtrsim 0.3 L^{*}$ galaxies across the supercluster, the bulk of fibres were then placed on galaxies in the range $13.0<W 1 \leq 14.7$. Finally, the remaining fibres are placed on lower mass galaxies in the range $14.7<W 1 \leq 15.5$, providing an effective survey limit of $\mathrm{W} 1=15.50 \mathrm{mag}$, corresponding to $\mathcal{M}=2.7 \times 10^{9} \mathrm{M}_{\odot}$ or $K *+3.7$. The use of $W 1$ magnitudes to place fibres should also ensure a simple and optimal analysis of supercluster galaxies based on new $K$-band photometry from our VISTA survey of the same ShaSS region carried out in 2014-15.

WISE W3 $(12 \mu \mathrm{m})$ photometry was also used to prioritize galaxies with active star formation ( $W 3<10.5 \mathrm{mag}$ ). This has the advantage of picking up dusty starbursts whose star formation is sufficiently obscured in the optical so that they appear on the red sequence (Haines, Gargiulo \& Merluzzi 2008; Wolf et al. 2009). Cluver et al. (2017) have shown that $12 \mu \mathrm{m}$ (W3) luminosities show a tight relation $(0.15$ dex scatter $)$ with the total infrared luminosity over 5 orders of magnitude, enabling $L_{W 3}$ to be used as a reliable star formation rate (SFR) estimator for both dwarf and giant galaxies. The W3 magnitudes of supercluster galaxies are compared to their $24 \mu \mathrm{m}$ fluxes obtained from our previous Spitzer/MIPS analysis of the Shapley supercluster core (Haines et al. 2011a) in Fig. 3. This demonstrates the tight relationship between the WISE W3 magnitudes and $24 \mu \mathrm{m}$ fluxes, and that the $W 3=10.5$ mag limit corresponds to an obscured SFR of $0.45 \mathrm{M}_{\odot} \mathrm{yr}^{-1}$ (dotted line). This SFR is low enough to include both star-forming and transitional 'green-valley' galaxies amongst the $\sim L *$ population (Haines et al. 2011b). Fig. 4 demonstrates this clearly, plotting WISE W3 magnitude versus $W 1$ for confirmed Shapley supercluster galaxies over the full ShaSS region. Two parallel diagonal sequences are seen, one at $W 3-W 1 \sim 0.5$ (lower dot-dashed line) consisting of passively-evolving galaxies, and a second sequence of star-forming galaxies at $W 3-W 1 \sim 4.0$ (upper dot-dashed line). There are few intermediate galaxies, enabling us to effectively separate the two populations about the line $W 3-W 1=2.25$ (dashed line), at least down to $W 1 \sim 14.0\left(\sim 0.1 L_{W 1}^{*}\right)$.

The software preparing targets for the $2 \mathrm{dF}$ fibre positioner allows the assigning of priorities to each target from 9 to 1 (highest to lowest). ${ }^{3}$ We assigned these priorities as indicated by the scheme shown in Table 1. Galaxies with existing redshift measurements were placed in the lowest priority level $(P=5)$. In summary this strategy enabled us to: (i) select galaxies according to their stellar

\footnotetext{
${ }^{3}$ The software for the preparation of such files is named 'configure', see http://www.aao.gov.au/science/software/configure.
} 


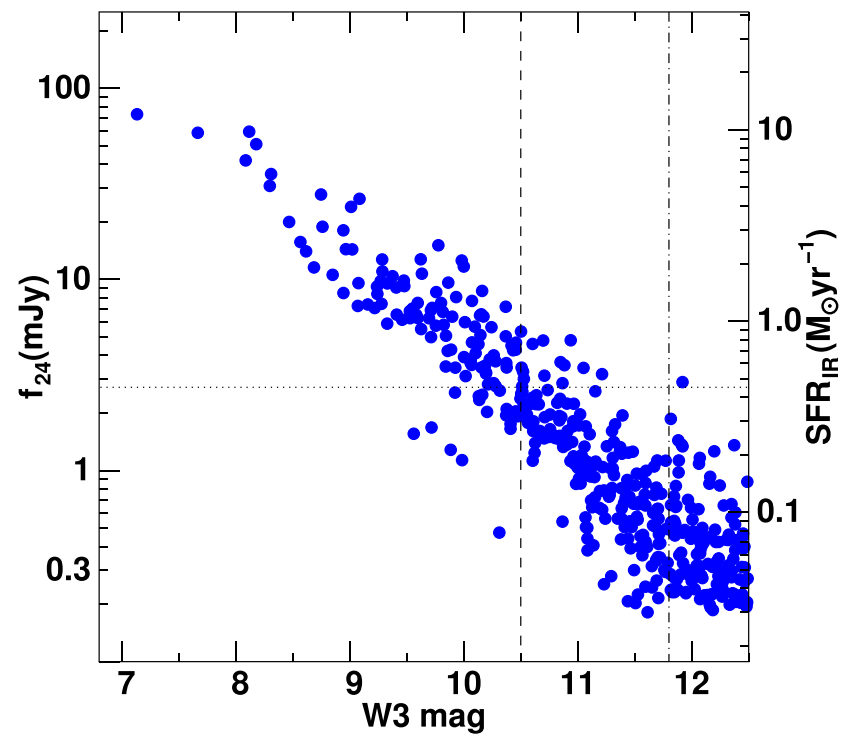

Figure 3. Relation between the WISE W3 magnitudes with the $24 \mu \mathrm{m}$ fluxes of confirmed Shapley supercluster galaxies detected in our previous Spitzer/MIPS analysis (Haines et al. 2011a). The right-hand axis shows the obscured SFRs derived using the $24 \mu \mathrm{m}$ SFR calibration of Leroy et al. (2008), which assumes a Kroupa IMF. The vertical dashed line shows the $W 3=10.5 \mathrm{mag}$ limit used in our redshift survey to prioritize star-forming galaxies. The dot-dashed line indicates the $5 \sigma$ limit of the WISE W3 photometry.

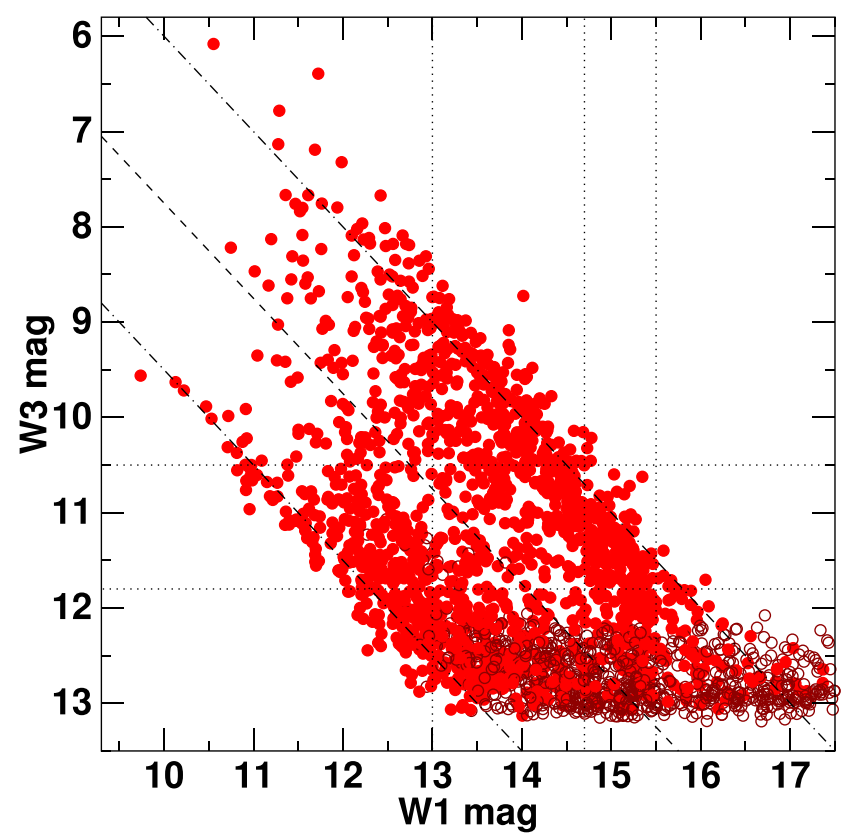

Figure 4. WISE magnitudes (W3 versus W1) of spectroscopic Shapley supercluster members over the full $21 \mathrm{deg}^{2}$ region. The three diagonal lines indicate $W 1-W 3=0.5,2.25$, and 4.0 from left to right. The lower dotted horizontal line shows the $5 \sigma \mathrm{W} 3$ limit, while the other dotted lines indicate the $W 1$ and $W 3$ magnitude limits adopted in Table 1. Open circles indicate galaxies not detected in $W 3$ at the $2 \sigma$ limit. The plot reveals two diagonal sequences, one at $W 1-W 3 \sim 0.5$ containing passive galaxies, and the main sequence of star-forming galaxies centred at $W 1-W 3 \sim 4.0$. A line midway between these two relations ( $W 1-W 3=2.25$; dashed line) is used to separate the two populations.
Table 1. Scheme of priorities adopted for fibre positioning.

\begin{tabular}{cccc}
\hline $\mathrm{P}$ & W1 limits & W3 limits & Comment \\
\hline 8 & $W 1<13.0$ & & $\mathcal{M}>2.7 \times 10^{10} \mathrm{M}_{\odot}$ \\
7 & $13.0<W 1<14.7$ & $W 3<10.5$ & $\mathrm{SFR}_{I R} \geq 0.45 \mathrm{M}_{\odot} \mathrm{yr}^{-1}$ \\
6 & $13.0<W 1<14.7$ & $W 3>10.5$ & $0.56-2.7 \times 10^{10} \mathrm{M}_{\odot}$, low SFR \\
5 & $14.7<W 1<15.5$ & & $2.7 \times 10^{9} \mathrm{M}_{\odot}<\mathcal{M}<$ \\
& & & $5.6 \times 10^{9} \mathrm{M}_{\odot}$ \\
\hline
\end{tabular}

masses; (ii) include both star-forming and transitional galaxies; (iii) uniformly map the local environment across the whole ShaSS region.

The centres of the pointings were determined using a simulated annealing algorithm in the same manner as Drinkwater et al. (2010). This allows the number of targets per two degree field to be optimized.

\section{OBSERVATIONS AND DATA REDUCTION}

Our spectroscopic observations were carried out using the AAOmega system (Smith et al. 2004a; Sharp et al. 2006) on the 3.9 m Anglo Australian Telescope, on the four nights of 2013 May 9-12. AAOmega consists of the $2 \mathrm{dF}$ fibre positioner (Lewis et al. 2002), linked to a stable, bench-mounted dual-beam spectrograph. The $2 \mathrm{dF}$ positioner can place 392 fibres, with 2 arcsec projected diameter, across a 2 deg diameter field of view. A further eight fibre bundles are used for guiding. Observations were carried out with $580 \mathrm{~V}$ and $385 \mathrm{R}$ gratings on the blue and red arm, respectively, covering the wavelength range $370-880 \mathrm{~nm}$ at a resolution of $\lambda / \Delta \lambda \sim 1300$. The integration time for each configuration was $1.5 \mathrm{~h}$, split into three $1800 \mathrm{~s}$ exposures.

A total of 14 AAOmega pointings were observed, the locations of which are shown in Fig. 2 by the numbered red circles. The seeing varied in the range $1.2-2.3$ arcsec with a median FWHM of $\sim 1.6$ arcsec. The success rate (the ratio of number of measured redshifts over the number of targets) was above 95 per cent for most pointings, with a peak of 99 per cent. For three pointings $(5,10$, and 14$)$ the success rate was initially low ( $<75$ per cent) which we ascribed to bad seeing or thin clouds. For these pointings we repeated the observations under better conditions reaching rates over 90 per cent. These duplicate observations provided us with a high number of repeated measurements of high quality, which we exploited to estimate the uncertainties (see Section 3.1). In each plate, 25 of the 392 fibres were dedicated to acquire sky spectra and several were dead and hence unavailable. A maximum of 363 fibres were placed on targets in a single configuration.

The data were reduced using the 2DFDR software (Lewis et al. 2002), which includes a Laplacian edge detection to remove incident cosmic rays. Redshifts were measured using the RUNZ code (adopted also in 2dFGRS, 2SLAQ, GAMA, and WiggleZ; see Colless et al. 2001; Cannon et al. 2006; Drinkwater et al. 2010; Driver et al. 2011). Further details of RUNZ are contained in Driver et al. (2011); here we summarize the pertinent points. The RUNZ code attempts to algorithmically determine a redshift from a given spectrum through cross-correlation with a range of template spectra that includes various stellar classes, QSOs, and galaxies ranging from highly starforming through to post-starburst and passive. As a second method, emission lines are fit with Gaussians and a search conducted for multiline matches. The code then displays the best fit to the operator (KAP), who performs an independent check of the correctness of the redshift. If it is incorrect, and it is obviously a different redshift, 
the user inputs the correct redshift (or the better template crosscorrelation) and updates the redshift. Each redshift is then assigned a quality $(Q)$ flag in line with GAMA (Driver et al. 2011) as follows:

$Q=4$ : The redshift is certainly correct.

$Q=3$ : The redshift is probably correct.

$Q=2$ : The redshift may be correct, but is insecure.

$Q=1$ : No redshift could be determined.

From the above, any redshift with $Q \geq 3$ can be used for science. Those assigned $Q=2$ were re-redshifted to determine if they could be placed in another category. A strong caveat in this process is that the assigned quality flag is subjective. All redshifts were therefore re-redshifted at least once (more in the case of $Q=2$ ) to ensure accuracy.

\subsection{The reliability and uncertainties of the redshift measurements}

In total we obtain spectra for 4186 unique sources, of which 4064 (including stars) have at least one reliable redshift measurement $(Q \geq 3)$. We have a final sample of 4027 galaxies that have reliable redshifts $(Q \geq 3)$, consisting of 957 galaxies within the broad redshift limits of the SSC $(0.03 \leq z<0.06)$, plus 59 confirmed foreground galaxies $(0.003 \leq z<0.03)$ and 3011 background galaxies out to $z=0.531$. Overall, reliable redshifts were obtained for 98.1 per cent of the targets observed.

As a result of reobserving three of the pointings, there are 1091 pairs of spectra from repeated observations of galaxies, each with reliable redshifts $(Q=4)$. The redshifts agree within $500 \mathrm{~km} \mathrm{~s}^{-1}$ for 1086 of these pairs, indicating a blunder rate of just 0.2 per cent. The implied mean internal error for each measurement is $51 \mathrm{~km} \mathrm{~s}^{-1}$, consistent with the typical pipeline errors from fitting the absorption lines $\left(\Delta V_{\mathrm{abs}} \sim 55 \mathrm{~km} \mathrm{~s}^{-1}\right)$. Where there are two or more redshift measurements of the same quality for a galaxy, its redshift is taken to be the mean of these measurements.

As an external validation, our redshifts are compared to those from the OmegaWINGS redshift survey (Moretti et al. 2017), which used AAOmega in 2013-15 to obtain spectra of galaxies from 33 low-redshift clusters, including three systems (A 3556, A 3558 and A 3560) within ShaSS. The redshifts agree within $500 \mathrm{~km} \mathrm{~s}^{-1}$ for 380 of the 384 objects in common (using only redshifts with $Q \geq 3$ ), implying a 99.0 per cent accuracy rate. The mean external error for each measurement is $60 \mathrm{~km} \mathrm{~s}^{-1}$, with no discernable systematic offset $\left(-2.7 \pm 3.1 \mathrm{~km} \mathrm{~s}^{-1}\right)$.

\subsection{Comparison and merging with literature data}

The Shapley supercluster has been repeatedly targetted by redshift surveys over the last $30 \mathrm{yr}$, some of which covered the full extent of the supercluster and others which focussed on the SSC or individual clusters.

The FLAIR Shapley-Hydra (FLASH) redshift survey (Kaldare et al. 2003) covered $700 \mathrm{deg}^{2}$ including the entire Shapley supercluster region (Fig. 1), targetting 3141 galaxies brighter than $b_{\mathrm{J}}=$ 16.7. FLASH measured redshifts for 355 galaxies within ShaSS, of which 252 are supercluster members. Drinkwater et al. (2004) measured redshifts for 710 galaxies with $R \leq 16.6$ over a $182 \mathrm{deg}^{2}$ region (similar to Fig. 1) with the FLAIR-II spectrograph on the UK Schmidt Telescope in Australia, of which 95 are covered by ShaSS. Bardelli et al. (1994, 1998b) carried out a spectroscopic survey of galaxies in the SSC with the Octopus multifibre spectrograph on the 3.6-m telescope at La Silla, measuring redshifts for 485 galaxies with $16.0 \lesssim b_{J} \lesssim 19.5$, all within ShaSS. This survey was widened to
Table 2. Offsets between $6 \mathrm{dF}$ redshifts and other sources.

\begin{tabular}{lcc}
\hline Source & $N_{\text {com }}$ & $\begin{array}{c}\left\langle\mathrm{V}_{h}-\mathrm{V}_{h}^{6 d F}\right. \\
\mathrm{km} \mathrm{s}^{-1}\end{array}$ \\
\hline Proust et al. (2006a) & 653 & $-31 \pm 121$ \\
Quintana et al. (1997) & 18 & $-24 \pm 50$ \\
NED $^{a}$ & 835 & $-4 \pm 48$ \\
Smith et al. (2004b) $^{\mathrm{b}}$ & 122 & $2 \pm 57$ \\
Smith et al. (2007) & 42 & $15 \pm 57$ \\
Drinkwater et al. (2004) & 72 & $-48 \pm 154$ \\
Kaldare et al. (2003) & 291 & $-14 \pm 151$ \\
Bardelli et al. (1998b) & 59 & $-3 \pm 96$ \\
Bardelli et al. (2000) & 16 & $73 \pm 141$ \\
Quintana et al. (1995) & 162 & $-49 \pm 314$ \\
Drinkwater et al. (1999) & 53 & $-50 \pm 158$ \\
\hline
\end{tabular}

ahttp://ned.ipac.caltech.edu/

${ }^{b}$ NOAO Fundamental Plane Survey (NFPS).

obtain redshifts for 442 intracluster galaxies with $17.0 \leq b_{\mathrm{J}} \leq 18.8$ (Bardelli et al. 2000), of which 111 lie within ShaSS. Quintana et al. $(1995,1997)$ measured redshifts for 795 galaxies across the Shapley supercluster (386 in ShaSS) to establish which Abell clusters belonged to the structure.

Smith et al. (2004b) targetted four clusters within ShaSS (A 3556 , A 3558, A 3560 and A 3562) with the Hydra multifibre spectrograph (40 arcmin diameter) on the 4-m telescope at CTIO, as part of the NOAO Fundamental Plane Survey, obtaining redshifts for 285 red sequence galaxies with $R<17$ (275 are SSC members). Smith et al. (2007) obtained spectra for 540 galaxies (448 members) in the SSC (A 3556, A 3558 and A 3562) with $R<18$ using AAOmega.

To merge our new redshifts with those already available from the above sources, we first have to account for possible systematic differences among different data sets, using the data in common. As we have targetted relatively faint galaxies $\left(\lesssim 0.4 L^{*}\right)$ and given low priority to those galaxies with existing redshifts, we often have few if any redshifts in common with an individual redshift survey, making a direct comparison unreliable. Instead, the redshifts from each of the above data sets were first homogeneized with the $6 \mathrm{dF}$ Galaxy Redshift Survey (6dFGRS; Jones et al. 2009). The 6dFGRS surveyed the entire Southern sky producing near-complete samples with $K \leq 12.65$ (Vega mag) taken from the 2MASS Extended Source Catalog (Jarrett et al. 2000), with additional targets to produce complete samples to $b_{\mathrm{J}}=15.60$ and $r_{\mathrm{F}}=16.75$. There are 3259 unique redshifts of galaxies over the full Shapley supercluster (region shown in Fig. 1) from the 6dFGRS, of which 752 are covered by our VST imaging survey. The 6dFGRS uses a modified version of the same software packages as used here, both for the data reduction and redshift measurements, ensuring consistency in the data handling. To homogeneize each redshift survey with the $6 \mathrm{dFGRS}$, the recession velocities of all the objects in common were compared. The results of these comparisons are summarized in Table 2, which lists the data sets, the number of objects in common with the 6dFGRS, the mean differences in heliocentric velocities and the relative $1 \sigma$ scatter between velocity measurements of individual sources. We subtracted the differences given in Table 2 from the literature redshifts and then merged them with the 6dFGRS catalogue to obtain a catalogue of literature redshifts in the 23 square degrees of the ShaSS survey.

The heliocentric velocities of the 292 objects in common between this combined catalogue of literature redshifts and our AAOmega data set were compared. A mean offset of $-27 \pm 85 \mathrm{~km} \mathrm{~s}^{-1}$ (literature-AAOmega) was obtained, which was subtracted from 
Table 3. Statistics of the merged redshift catalogue.

Total number of redshifts in the whole ShaSS area

New redshifts in 21 square degrees

Literature redshifts in 21 square degrees

Redshifts in common between literature and AAOmega

Literature redshifts in ShaSS fields \# 19 and 20

Additional redshifts from OmegaWINGS

the literature data before merging the two data sets. Finally, the redshifts from the OmegaWINGS survey (Moretti et al. 2017) were added and merged, without further adjustment. The statistics of the final catalogue is given in Table 3, confirming that our AAOmega survey increased the number of redshifts in its area by a factor 2.09.

\subsection{Completeness of all available redshifts}

As described in Section 2.2, the AAOmega survey aims to obtain redshifts for a highly complete sample of galaxies with $i \leq 18.0$ and $W 1 \leq 15.5$ over the ShaSS area. There are 6008 galaxies satisfying these selection criteria across the 21 square degrees covered. Reliable redshifts were obtained for 4027 of these 6008 galaxies through our AAOmega observations. After including the existing redshifts from the literature, we now have redshifts for 5689 of the galaxies (with $i \leq 18.0$ and $W 1 \leq 15.5$ ), meaning an overall completeness of 94.7 per cent over the 21 square degree survey region. The completeness is uniformly high across the survey, being above 92 per cent in 19 out of the 21 VST fields, falling to $\sim 85$ per cent in fields 10 and 22. The highest completeness levels ( 98.7 per cent) are seen in the densest fields (fields 8 and 13).

Massive galaxies were prioritized when placing fibres in an attempt to obtain redshifts for all $\gtrsim L^{*}$ galaxies in the supercluster. This objective was achieved, with 100 per cent redshift completeness for the 763 galaxies with $W 1<13.1$ mag and the 1025 galaxies with $i_{\mathrm{AB}}<15.84 \mathrm{mag}$. Reliable redshifts were also obtained for all 407 galaxies with $W 3<9.75 \mathrm{mag}\left(\mathrm{SFR}_{\mathrm{IR}}>0.9 \mathrm{M}_{\odot} \mathrm{yr}^{-1}\right)$. The redshift survey is also 98.0 per cent complete for the 1670 galaxies with $W 3<11.0 \mathrm{mag}$, ensuring a complete census of obscured star-formation across the whole ShaSS region down to $\mathrm{SFR}_{\mathrm{IR}} \sim 0.3 \mathrm{M}_{\odot} \mathrm{yr}^{-1}$.

Fig. 5 shows the $W 1$ versus $i$-band magnitudes of galaxies in the ShaSS region, with red (orange) points marking Shapley supercluster members with redshifts from our AAOmega survey (the literature), blue (green) points indicating confirmed background (foreground) galaxies, and grey points galaxies that still lack a redshift. This shows that effectively our survey is targetting supercluster members down to $W 1=15.5$, the $i=18.0$ cut primarily excluding background galaxies.

The orange (dark red) histograms in the lower panels show the redshift completeness of our survey as a function of $i$-band and $W 1$ magnitudes, including (excluding) those redshifts coming from the literature. This reaffirms that we remain 100 per cent complete to $i$ $=15.75$ and $W 1=13.0$. The completeness then drops only slowly to 90 per cent in the last magnitude bin before our survey limits $(i=$ $18.0, W 1=15.5$ ). At bright magnitudes, only 30 per cent of galaxies were observed by us with AAOmega, as the remainder already had known redshifts, but our survey becomes the dominant source of redshifts at $i>16.5$ and $W 1>14.0$.
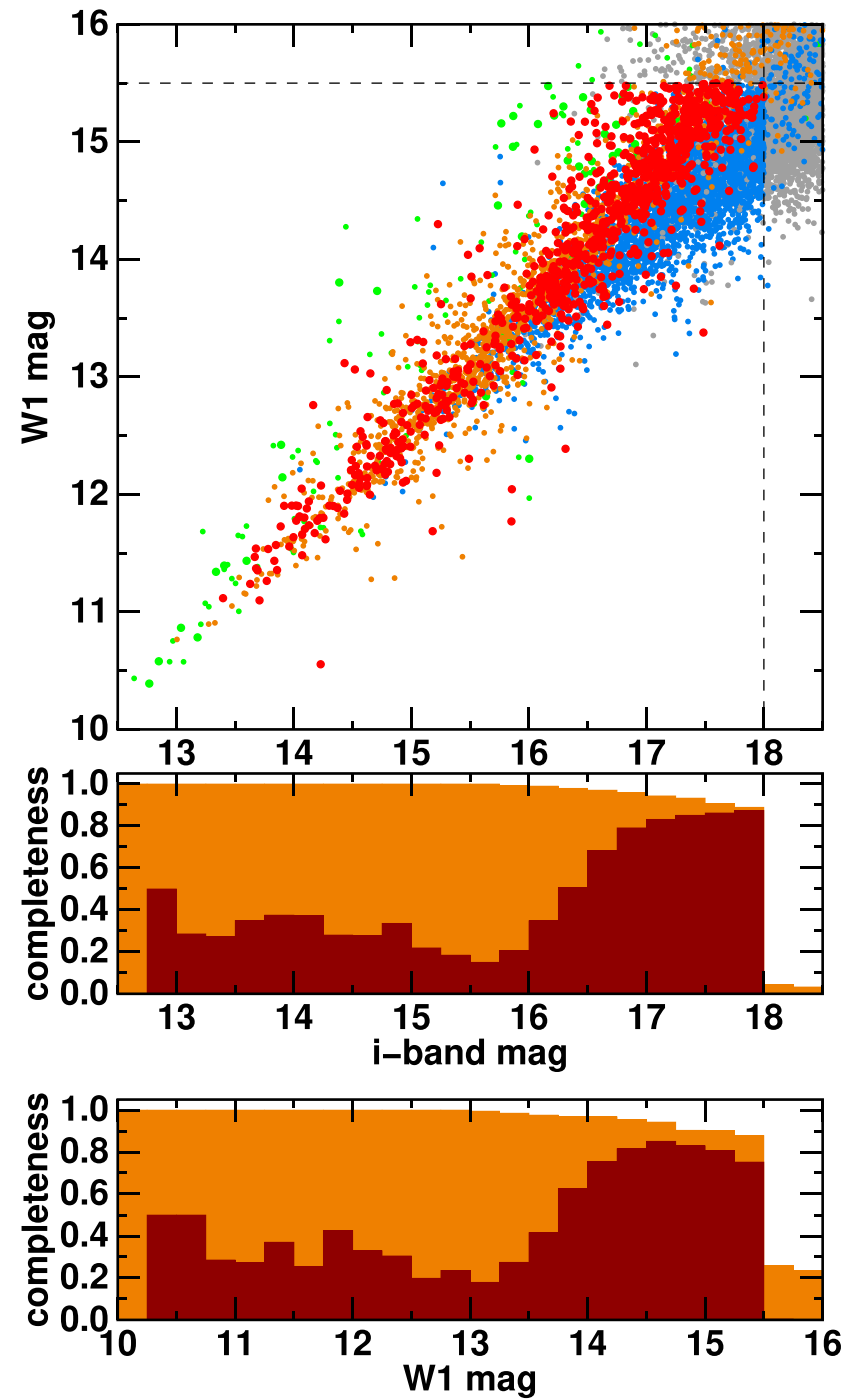

Figure 5. (top panel) WISE $W 1$ mag versus VST $i$-band magnitudes of galaxies in the $21 \mathrm{deg}^{2}$ AAOmega survey region. Confirmed Shapley supercluster members with redshifts obtained from our AAOmega survey (the literature) are shown as red (orange) symbols, while blue (green) points mark galaxies whose redshifts place them behind (in front of) the supercluster. Grey symbols indicate galaxies with no known redshift. (lower panels) The redshift completeness over the $21 \mathrm{deg}^{2}$ region as a function of $i$-band and $W 1$ magnitude (orange histogram). The dark red histogram shows the fraction of galaxies with redshifts from our AAOmega survey alone.

\section{AN UPDATED MAP OF THE SHAPLEY SUPERCLUSTER}

Fig. 6 shows the overall distribution of galaxy velocities $(c z)$ across the ShaSS survey region (blue histogram), combining our new AAOmega redshifts with those from previous studies. The vertical scale is set to highlight the structures beyond the Shapley supercluster out to $64000 \mathrm{~km} \mathrm{~s}^{-1}$, with the most evident peaks at $27500,35000,40000,43000$, and $53000 \mathrm{~km} \mathrm{~s}^{-1}$. The overdensity of galaxies in the SSC is so great, that the associated peak in the velocity distribution reaches 262 galaxies per bin, $\sim 5 \times$ higher than any of the other peaks in the distribution. We thus replot the velocity distribution of galaxies in the velocity range of the SSC (11 000$17500 \mathrm{~km} \mathrm{~s}^{-1}$ ) after scaling down by a factor three (red histogram). Beyond the SSC, there is a $4000 \mathrm{~km} \mathrm{~s}^{-1}$ wide gap in the distribu- 


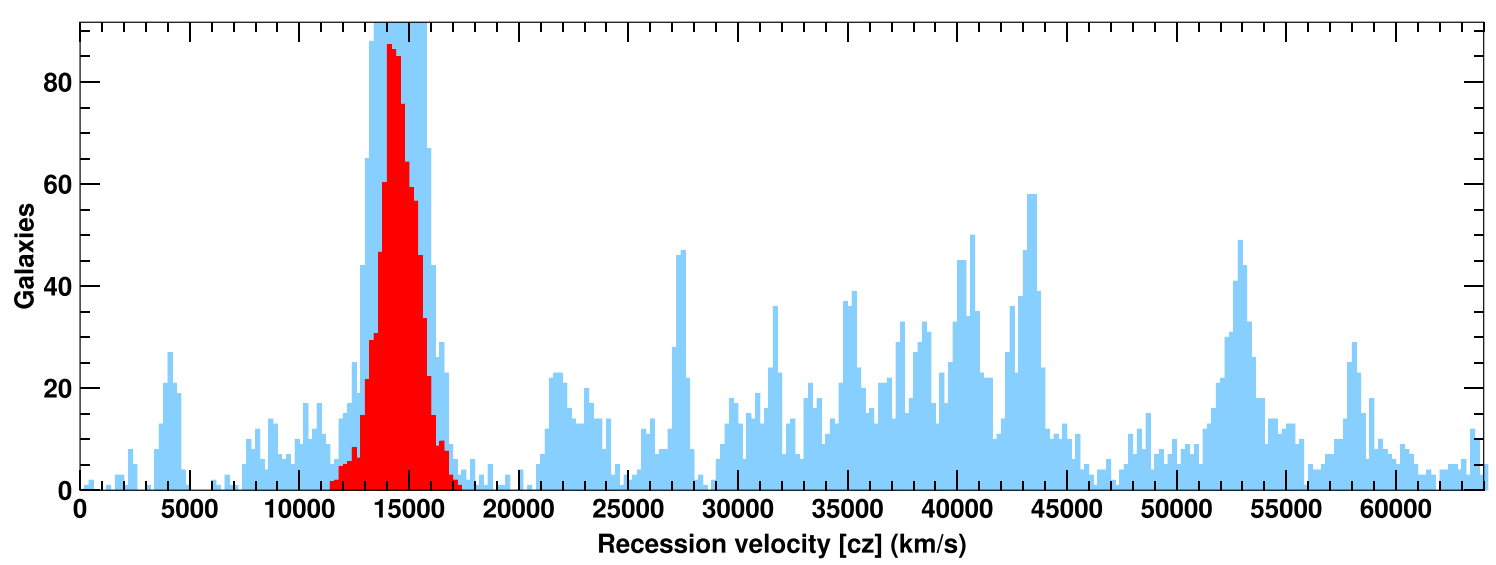

Figure 6. Velocity $(c z)$ distribution of galaxies across the ShaSS region (light blue histogram) over the velocity range $V_{h}=0-64000 \mathrm{~km} \mathrm{~s}^{-1}$ and bin width of $200 \mathrm{~km} \mathrm{~s}^{-1}$. The red histogram shows the velocity distribution of supercluster galaxies $\left(10500-17500 \mathrm{~km} \mathrm{~s}^{-1}\right)$, scaled down by a factor 3 to fit on the plot.

tion, where virtually no galaxies are found in the velocity range $17000-21000 \mathrm{~km} \mathrm{~s}^{-1}$. In the foreground, a peak at $4000 \mathrm{~km} \mathrm{~s}^{-1}$ is due to a wall of galaxies within the Hydra-Centaurus supercluster that traverses the ShaSS region (Radburn-Smith et al. 2006; Huchra et al. 2012), including the embedded group centred on NGC 5153.

It is worth comparing this velocity histogram with that presented by Proust et al. (2006a, their Fig. 2) for galaxies over the wider Shapley supercluster region $\left(285 \mathrm{deg}^{2}\right)$. Notably we observe a much deeper void on the far side of the SSC, as we are focussed on the densest, central region. Proust et al. (2006a) also observe a second peak at $\sim 11000 \mathrm{~km} \mathrm{~s}^{-1}$, due to the so-called 'Front Eastern Wall' (Quintana, Carrasco \& Reisenegger 2000) of the Shapley supercluster, which is largely absent in our velocity distribution. This wall includes the clusters A 3572 and A 3575 (Fig. 1), but it shows little sign of extending through the ShaSS region.

\subsection{Cone diagrams}

Fig. 7 shows the resulting distribution of galaxies towards the Shapley supercluster as a cone diagram in right ascension. The supercluster itself can be seen as the dense clump of galaxies at $\sim 14000 \mathrm{~km} \mathrm{~s}^{-1}$, but filamentary structures can be seen to extend across the whole ShaSS field of view, to $z \sim 0.2$ and beyond. The increased depth (to $i=18 \mathrm{mag}$ ) of the new AAOmega survey has enabled the background structures to be mapped in much more detail than previous surveys (e.g. Drinkwater et al. 2004, which reached $R=16.6$ ). A detailed knowledge of the backround structures will also be vital for our ongoing weak lensing analysis (Merluzzi et al. 2015), enabling mass overdensities within the supercluster to be distinguished from those due to background clusters.

One example is the clump of galaxies at $z \sim 0.103$, RA 198.5 , which can be identified with the cluster AS 0724b $(z=0.101)$ in Proust et al. (2006a). While there is no obvious central concentration of galaxies containing a dominant BCG, we do see a significant filamentary structure extending across the full Western edge of the ShaSS-AAOmega survey (RA 198.7, $-34<$ Dec $<-31$ ), containing $\sim 150$ galaxies with $0.097<z<0.109$. This structure then likely connects to the clusters AS $0725(\alpha=198.54, \delta=$ $-30.20, z=0.1067)$ and Abell $3546(\alpha=198.26, \delta=-29.98, z$ $=0.1065$ ), which lie just beyond the region covered by AAOmega, forming the supercluster SSCC 259 identified by Chow-Martínez et al. (2014).
The sharp peak at $27500 \mathrm{~km} \mathrm{~s}^{-1}$ in Fig. 6 does not contain any single dense concentration of galaxies in the sky, but rather a series of filamentary structures extending across the full ShaSS region (Fig. 7), seemingly connecting to Abell $3545(z=0.09)$ which lies ( $\alpha=197.98, \delta=-34.08$ ) just beyond the SW corner of ShaSS.

Fig. 8 shows the same distribution of galaxies towards the Shapley supercluster as cone diagrams in RA (upper panel) and Dec (lower panel), but limited to velocities below $24000 \mathrm{~km} \mathrm{~s}^{-1}$ to focus on the supercluster itself and the void immediately behind it. The upper and lower velocity limits of the supercluster are sharply defined in many regions in these two plots, enabling simple membership classification. For example, while the supercluster densely fills the region $0.04<z<0.055$ and $\mathrm{RA}>202^{\circ}$, there are no galaxies in the $0.055<z<0.0625$ range immediately beyond it, a velocity gap of $2200 \mathrm{~km} \mathrm{~s}^{-1}$. A similar clear gap is seen at 12000 $12800 \mathrm{~km} \mathrm{~s}^{-1}$ and $\mathrm{RA}<201^{\circ}$. A precise separation of supercluster members and non-members is aided significantly by the void on the far side, which extends for $4000 \mathrm{~km} \mathrm{~s}^{-1}$ beyond the supercluster (to $z \sim 0.07$ ) over the entire ShaSS region. Such sharp delineations and adjacent gaps in redshift space are expected for galaxies infalling into virialized structures, producing characteristic caustic features (Diaferio \& Geller 1997). On the far side of the void, a group of $\sim 16$ galaxies is apparent at $z=0.077$, centred on a massive elliptical at $\alpha=13: 24.53 .4, \delta=-29: 17: 56$.

The situation on the near side of the supercluster is complicated by the corkscrew-like structure spiraling out from the low-velocity side of A 3558 (RA $202^{\circ}$, Dec $\sim 31.5^{\circ}, V_{h} \sim 12000 \mathrm{~km} \mathrm{~s}^{-1}$ ) down to velocities of $\sim 7500 \mathrm{~km} \mathrm{~s}^{-1}$. At $11000 \mathrm{~km} \mathrm{~s}^{-1}$ this spiral reaches the eastern limit of our survey region, where it then connects to a sheet of galaxies at $\sim 11000 \mathrm{~km} \mathrm{~s}^{-1}$ that extends East to Abell 3571 $\left(\mathrm{RA}=206.9^{\circ}\right)$ and beyond (Drinkwater et al. 2004).

\subsection{Creating a density map of the Shapley supercluster and identifying groups of galaxies}

To assemble a density map of galaxies across the Shapley supercluster, we apply a variant of the adaptive kernel estimator (Silverman 1986; Pisani 1993, 1996) where each galaxy $i$ within the pre-determined velocity limits of the Shapley supercluster is represented with an adaptive Gaussian kernel $\kappa_{i}(\mathbf{x}, z)$ in redshift space. Following Haines et al. (2007), the width of the kernel is fixed in the radial direction to $500 \mathrm{~km} \mathrm{~s}^{-1}$, while the widths in the transverse directions are given by $\sigma_{i}=(8 / 3)^{1 / 2} D_{3}$, where $D_{3}$ is the distance 


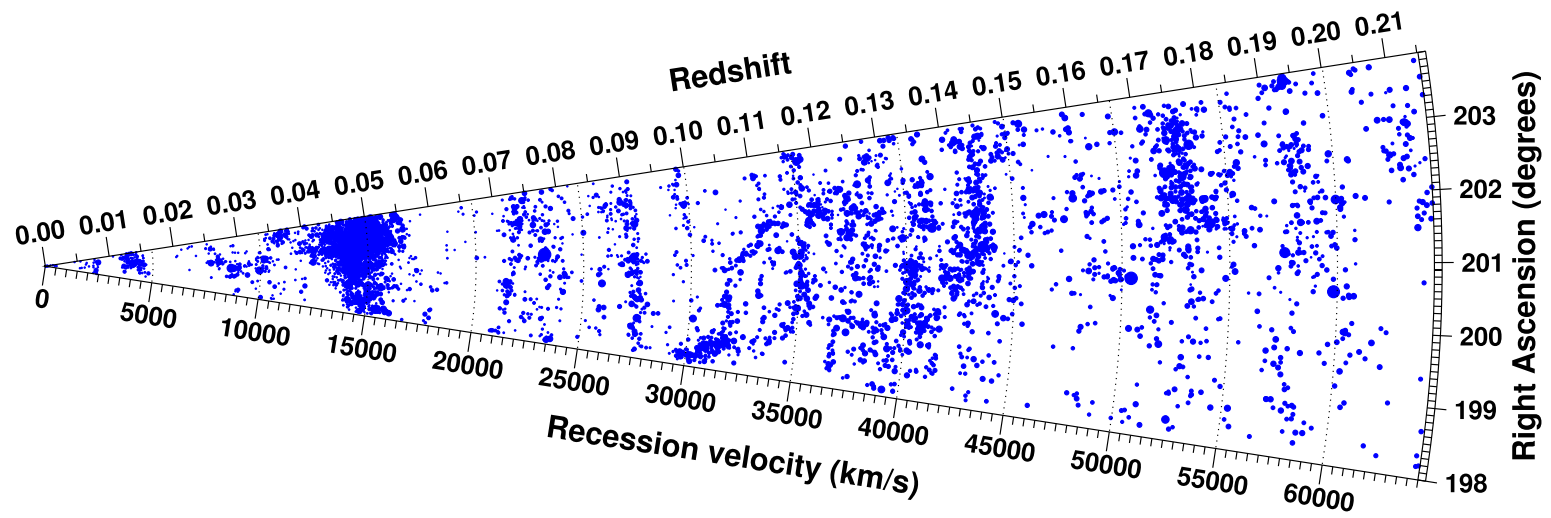

Figure 7. Cone velocity diagram in right ascension $\left(198^{\circ} \leq \mathrm{RA} \leq 204^{\circ}\right)$ for galaxies with measured redshifts in the ShaSS region out to $V_{\mathrm{h}}=64000 \mathrm{~km} \mathrm{~s}^{-1}$. The symbol size indicates the $i$-band luminosity of each galaxy.

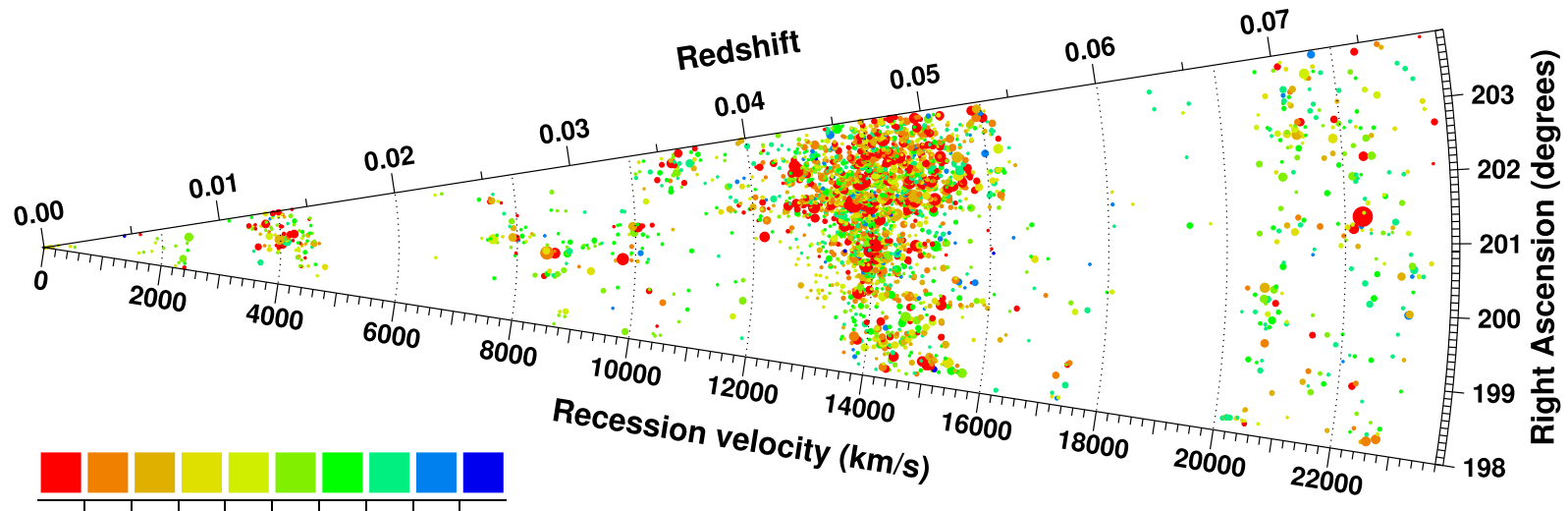

0.51 .01 .52 .02 .53 .03 .54 .04 .5

W1 - W3 (mag)

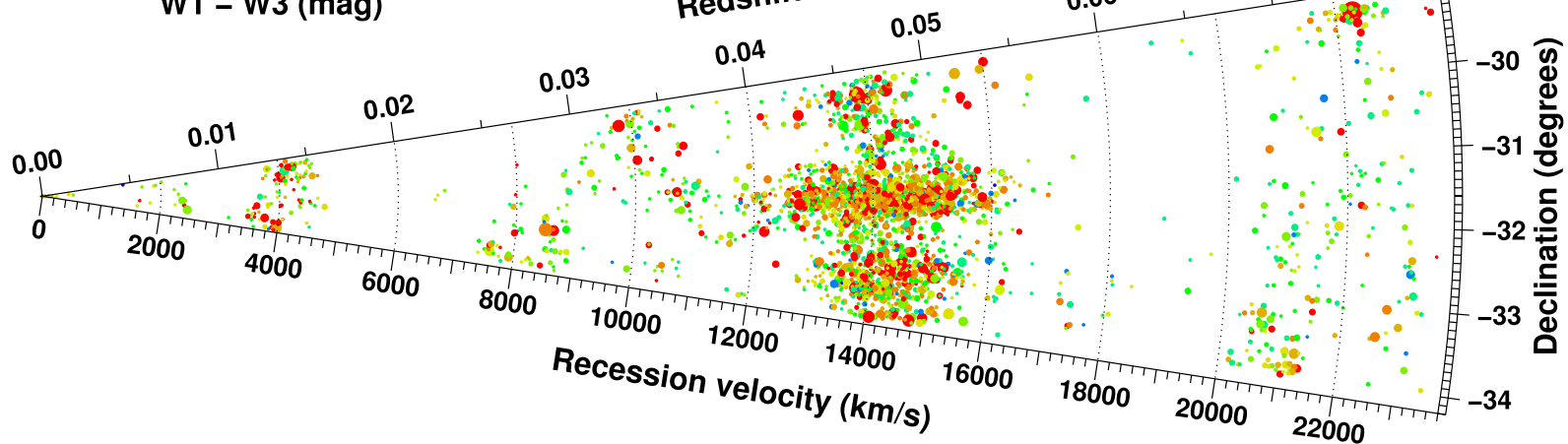

Figure 8. Cone velocity diagram in right ascension $\left(198^{\circ} \leq \mathrm{RA} \leq 204^{\circ}\right.$; upper panel) and declination $\left(-34.2^{\circ} \leq\right.$ Dec $\leq-29^{\circ}$; lower panel) for galaxies with measured redshifts in the ShaSS region inside $V_{\mathrm{h}}=24000 \mathrm{~km} \mathrm{~s}^{-1}$. The symbol size and colour indicate the $i$-band luminosity and $W 1-W 3$ colour of each galaxy.

of the third nearest neighbour within $500 \mathrm{~km} \mathrm{~s}^{-1}$ of galaxy $i$. The choice of $D_{3}$ was made to maximize the sensitivity of the density estimator to poor groups containing as few as four galaxies. The choice of the method and kernel dimensions is designed to resolve the galaxy's environment on the scale of its host DM halo $(0.1-1 \mathrm{Mpc})$ for those galaxies within groups, while for galaxies in isolated field regions, the local density is estimated by smoothing over its 5-10 nearest neighbours. For each galaxy $i$, the local galaxy density is defined as

$\rho_{i}(\mathbf{x}, z)=\sum_{j} \eta_{j} \exp \left\{-\frac{1}{2}\left[\left(\frac{D_{i j}}{\sigma_{j}}\right)^{2}+\left(\frac{v_{i}-v_{j}}{500 \mathrm{~km} \mathrm{~s}^{-1}}\right)^{2}\right]\right\}$ where $\eta_{j}=w_{j}(2 \pi)^{-3 / 2} \sigma_{j}^{-2}$ is the normalization factor, $D_{i j}$ is the projected distance between galaxies $i$ and $j$, and $v_{i}$ is the recession velocity of galaxy $i$. We account for those supercluster galaxies lacking redshifts by weighting each galaxy by the inverse probability of it being observed spectroscopically, following Norberg et al. (2002). All galaxies that could have been targetted with AAOmega (i.e. having $i<18.0$ and $\mathrm{W} 1<15.5$ ) are first given equal weight (1.0). For each galaxy lacking a redshift, its weight is transferred equally to its 10 nearest neighbouring galaxies with known redshift that also had the same priority level in our targetting strategy (Table 1). Each galaxy with a redshift is left with a weight $w_{j}$ equal to the inverse of the local spectroscopic completeness $\left(\mathcal{C}^{-1}\right)$. 
The adaptive kernel estimator can be used as a group-finder (e.g. Bardelli et al. 1998a; Haines et al. 2006), with groups and clusters identified as local maxima in the 3D galaxy density field $\rho(\mathbf{x}, z)$. The peak associated to each galaxy is determined by following a path of maximal gradient uphill starting from each galaxy until a local maximum is reached, enabling the members of each group to be ascertained. This would imply that all galaxies belong to a group, and so additional steps are required to limit group membership to galaxies within $r_{200}$ and appropriate velocity limits, identify and exclude isolated galaxies.

Fig. 9 shows the resultant bidimensional density map of confirmed supercluster galaxies over the full ShaSS region, calculated as

$\rho(\mathbf{x})=\sum_{i} \frac{w_{i}}{2 \pi \sigma_{i}^{2}} \exp \left[-\frac{1}{2}\left(\frac{\left|\mathbf{x}-\mathbf{x}_{\mathbf{i}}\right|}{\sigma_{i}}\right)^{2}\right]$,

where $\mathbf{x}_{\mathbf{i}}$ is the position of galaxy $i$. Its objective is to characterize the local environment of each galaxy in the supercluster, and complements the map of stellar mass density presented in Merluzzi et al. (2015).

The yellow circles mark the $r_{200}$ radii of the 11 known X-ray clusters, as calculated in Section 5. This highlights the difficulty in separating the clusters and substructures in the SSC, with the $r_{200}$ radii of the five clusters overlapping each other. The cluster SC 1327-312 in particular lies almost entirely within the virial radius of Abell 3558. The galaxy overdensity in the SSC gets steadily wider in the $\mathrm{N}-\mathrm{S}$ direction moving West from A 3562 to A 3558. There is then a sharp N-S edge just beyond A 3558 where the galaxy density drops rapidly, except along a narrow filament extending through A 3556 to a group located on its Western edge (labelled A3556$\mathrm{W})$. This $\mathrm{N}-\mathrm{S}$ edge likely marks the boundary where galaxies have passed through A 3558 and are rebounding out, while A 3556 is still on its first infall towards A 3558. A weaker filamentary structure extends West from A 3556 through A 3552 before bending South to connect up to AS 0724, AS 0726, and Abell 3554.

A filamentary structure can be seen heading North from the SSC, extending $\sim 2^{\circ}(\sim 7 \mathrm{Mpc})$ up to reach Abell 3559 . Two galaxy groups are embedded in the filament connecting A 3559 to the SSC.

Abell 3560 is the most massive of the other clusters in ShaSS but outside the SSC. It has two distinct substructures within $r_{200}$, one to the North and one to the West. There is no apparent structure connecting A 3560 directly to the SSC along the shortest path (North to SC 1329-317). The most likely direction for any connecting structure is NW towards A 3558.

Bardelli et al. (1998a) used a similar algorithm to detect substructures in the $3 \mathrm{D}$ distribution of galaxies within the SSC, revealing its extraordinary dynamical complexity, finding eight subclumps within Abell 3558 and four within A 3556. Many of the clumps and secondary peaks in the SSC apparent in Fig. 9 match the groups identified by the 3D analysis of Bardelli et al. (1998a) and are labelled in the figure by their original IDs (e.g. T49, T598).

The local galaxy number density $\rho(\mathbf{x})$ is indicated in Fig. 9 both by the colour shading and the grey isodensity contours. The contours are logarithmically spaced with 0.1 dex separation, with the outermost contour corresponding to a number density of $2.0 \mathrm{gals}_{\mathrm{Mpc}}^{-2}$. To compare this with that expected for an average field region at the same redshift, we select galaxies with the same magnitude and velocity limits from the three equatorial fields (G09, G12, G15) of the GAMA survey (Driver et al. 2011). The GAMA II main survey provides a highly complete (98.5 per cent; Liske et al. 2015) spectroscopic sample of $r<19.8$ galaxies across these three fields (i.e. deeper than our $i=18.0$ limit), as well as optical ugriz and WISE near-infrared photometry, made available through the third data release (GAMA-DR3 Baldry et al. 2018). There are 2218 galaxies with $W 1<15.5, i<18.0$, and $10500<v<17000 \mathrm{~km} \mathrm{~s}^{-1}$ across the $180 \mathrm{deg}^{2}$ region covered by the three GAMA fields, corresponding to $1.07 \pm 0.25 \mathrm{gals} \mathrm{Mpc}^{-2}$. The lowest contour in Fig. 9 thus marks a local galaxy density which is double that is seen in the field, i.e. all regions of ShaSS are overdense.

\subsection{The connectivity of the Shapley supercluster}

In the previous section we produced a galaxy density map (Fig. 9), which indicates the relative location of the clusters on the sky, and possible connecting structures in the form of overdense regions. We now seek to establish if, and how each of the clusters are kinematically connected to one another.

Fig. 10 plots recession velocity $(c z)$ against right ascension (RA) for galaxies within the overall velocity limits of the Shapley supercluster (10 500-17 $500 \mathrm{~km} \mathrm{~s}^{-1}$ ) across the ShaSS survey region, after splitting into five approximately degree-wide declination strips. This slicing enables the structures within the survey volume to be separated and isolated. Fig. 11 shows the recession velocities of the same supercluster galaxies, but plotted against Declination after splitting into five strips of RA. Here, we assume that variations in velocity mostly represent differences in LOS peculiar velocities within the supercluster rather than major differences in distance, so that a simple vertical axis is more appropriate than a wedge diagram.

These two plots allow the supercluster to be traced across the inter-cluster regions over the full extent of the survey area, the identification of filaments connecting clusters, breaks, or gaps in the structure, as well as velocity gradients in the supercluster that could indicate either real physical variations in the distances to the supercluster from one direction to another, or infall velocities due to the (spherical) collapse of the supercluster onto the central mass concentration (Reisenegger et al. 2000).

In each of the five declination slices of Fig 10, a continuous ribbon of galaxies traverses the full extent of the ShaSS survey region in RA with no apparent gaps. There are also no significant velocity gradients when moving in the RA direction, with the ribbon of galaxies always found at $\sim 14500 \mathrm{~km} \mathrm{~s}^{-1}$. While the velocity range covered by supercluster galaxies is wide in the SSC $\left(\sim 5000 \mathrm{~km} \mathrm{~s}^{-1}\right.$; central panel) and the vicinity of the known clusters, the ribbon becomes rather narrow in the regions away from any cluster. The velocity dispersion of supercluster members in these regions (e.g. top panel, RA < 201.9) falls to $\sim 200 \mathrm{~km} \mathrm{~s}^{-1}$.

Fig. 11 shows that these continuous structures extend also in the declination direction across the full ShaSS area. The distribution of galaxies in the central panel is reminiscent of a crosssection through a spiral galaxy, with a flat disk extending across the full width and a central bulge around A 3556 where the velocity dispersion increases. Only in the bottom two panels are significant velocity gradients apparent, with the structure connecting A 3562 and A 3560 'sagging' well below (at $\sim 13000 \mathrm{~km} \mathrm{~s}^{-1}$ ) the central redshifts of each cluster $\left(\sim 14700 \mathrm{~km} \mathrm{~s}^{-1}\right)$, while galaxies arriving into A 3562 from the North have recession velocities of $\sim 15500 \mathrm{~km} \mathrm{~s}^{-1}$.

Figs 10 and 11 demonstrate that the Shapley supercluster consists of a coherent sheet of galaxies at $\sim 14500 \mathrm{~km} \mathrm{~s}^{-1}$ that permeates the full extent of the ShaSS survey region with no gaps or holes. The 11 known X-ray clusters are all embedded within this sheet and hence physically interconnected.

Either side of the sheet are wide regions in velocity space $\left(>1000 \mathrm{~km} \mathrm{~s}^{-1}\right)$ that are entirely devoid of galaxies. These are sig- 


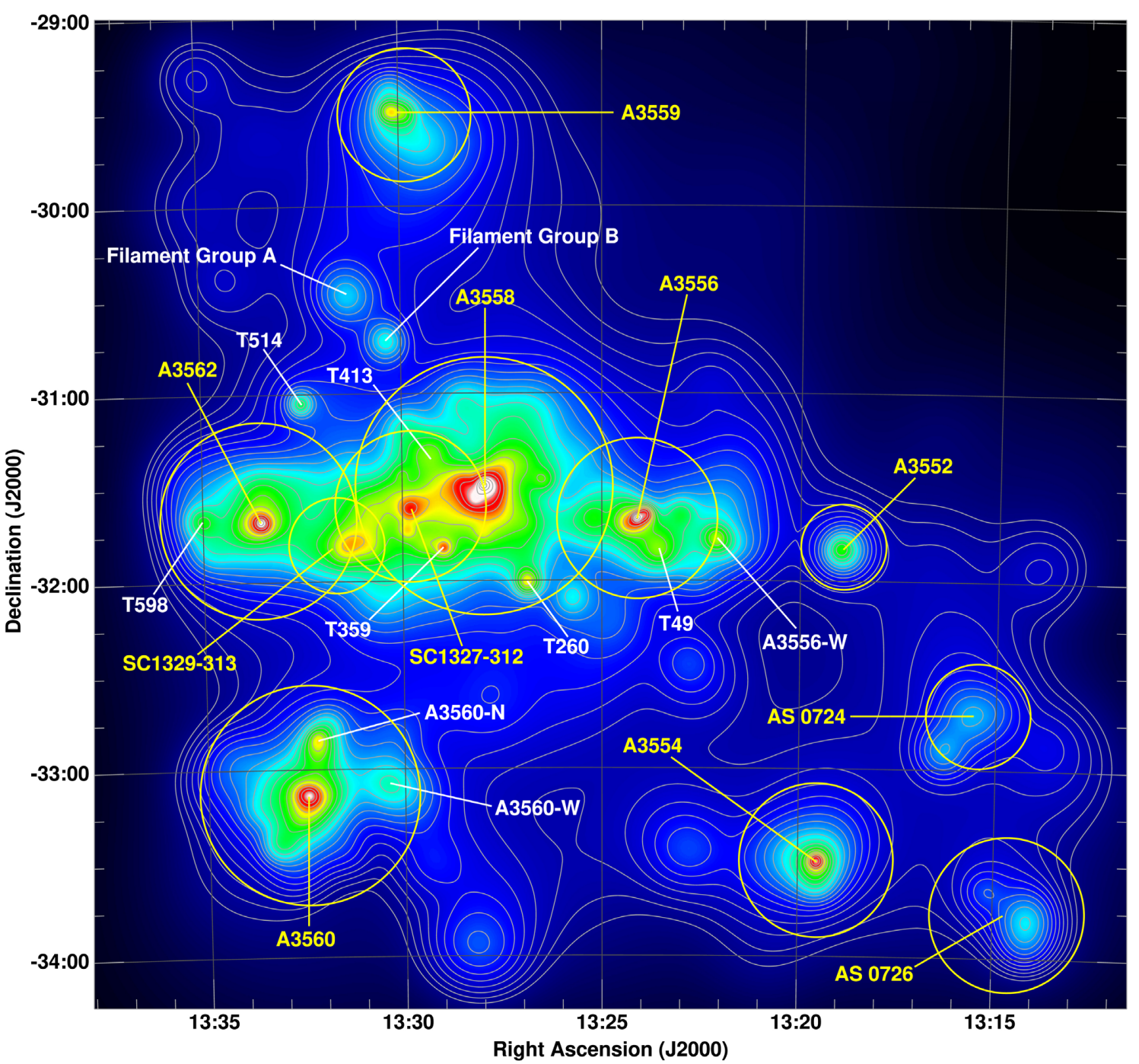

Figure 9. The bidimensional distribution of Shapley supercluster galaxies across the ShaSS survey region. The grey isodensity contours are logarithmically spaced with 0.1 dex separation. The 11 known clusters are labelled and their $r_{200}$ radii shown by yellow circles. Other groups discussed in the text are labelled in white. Groups from the 3D substructure analysis of the SSC by Bardelli et al. (1998a) are highlighted. These are labelled with T followed by a three-digit number (e.g. T598) as in their Table 2.

nificantly underdense regions, even with respect to the field. In the GAMA survey we would expect to find $\sim 4$ galaxies per $\mathrm{deg}^{2}$ in the velocity range $10500-13000 \mathrm{~km} \mathrm{~s}^{-1}$ (the lower third of each panel in Fig. 10). This supports the view that the sheet covering the entire ShaSS region has detached from the cosmic Hubble flow, along with all 11 embedded clusters, and is in the process of collapsing inwards towards Abell 3558 under the effects of its own gravity, as asserted by Reisenegger et al. (2000), Ettori et al. (1997) and de Filippis et al. (2005).

Fig. 12 plots the distribution of supercluster members (red points) and non-members (light blue points) in the caustic diagram (line-ofsight velocity, $c z$, versus projected distance from the centre of Abell 3558). The supercluster members are confined within a well-defined 'trumpet'-shaped region that narrows with increasing cluster-centric distance, as expected for galaxies infalling and subsequently orbiting a massive virialized structure (Regös \& Geller 1989; Dünner et al. 2006; Haines et al. 2015). There is a sharp boundary between the interior of this 'trumpet'-shaped region where the phase-space density of galaxies is high, and outside where few if any galaxies are observed. Notably, both in the supercluster core $(r<0.5 \mathrm{deg})$ and towards the outer regions of the survey $(r>2.7 \mathrm{deg})$ there are no galaxies within $1000 \mathrm{~km} \mathrm{~s}^{-1}$ of the caustics, making their definition unambiguous. The foreground galaxies with velocities $\sim 10-12000 \mathrm{~km} \mathrm{~s}^{-1}$ lie along the Eastern edge of the ShaSS region (bottom panel of Fig. 11), forming part of the 'Front Eastern Wall' (Quintana et al. 2000).

The trumpet shape of the caustic profile reflects the dynamics and infall velocities of galaxies in the infall regions of a massive cluster (Regös \& Geller 1989), and its amplitude reflects the escape velocity from the cluster at that radius (Diaferio \& Geller 1997). The caustics enclose all galaxies within the turn-around radius, i.e. those which have detached from the Hubble flow (Diaferio 1999). This compresses their distribution in the velocity direction of Fig. 12 at large radii, and empties the regions of phase-space immediately outside the caustics (Regös \& Geller 1989). The ability to trace the caustic profile right to the survey boundary indicates that the entire region is gravitationally bound and in the process of collapse, since the width of the caustic should fall to zero at the turnaround radius. 


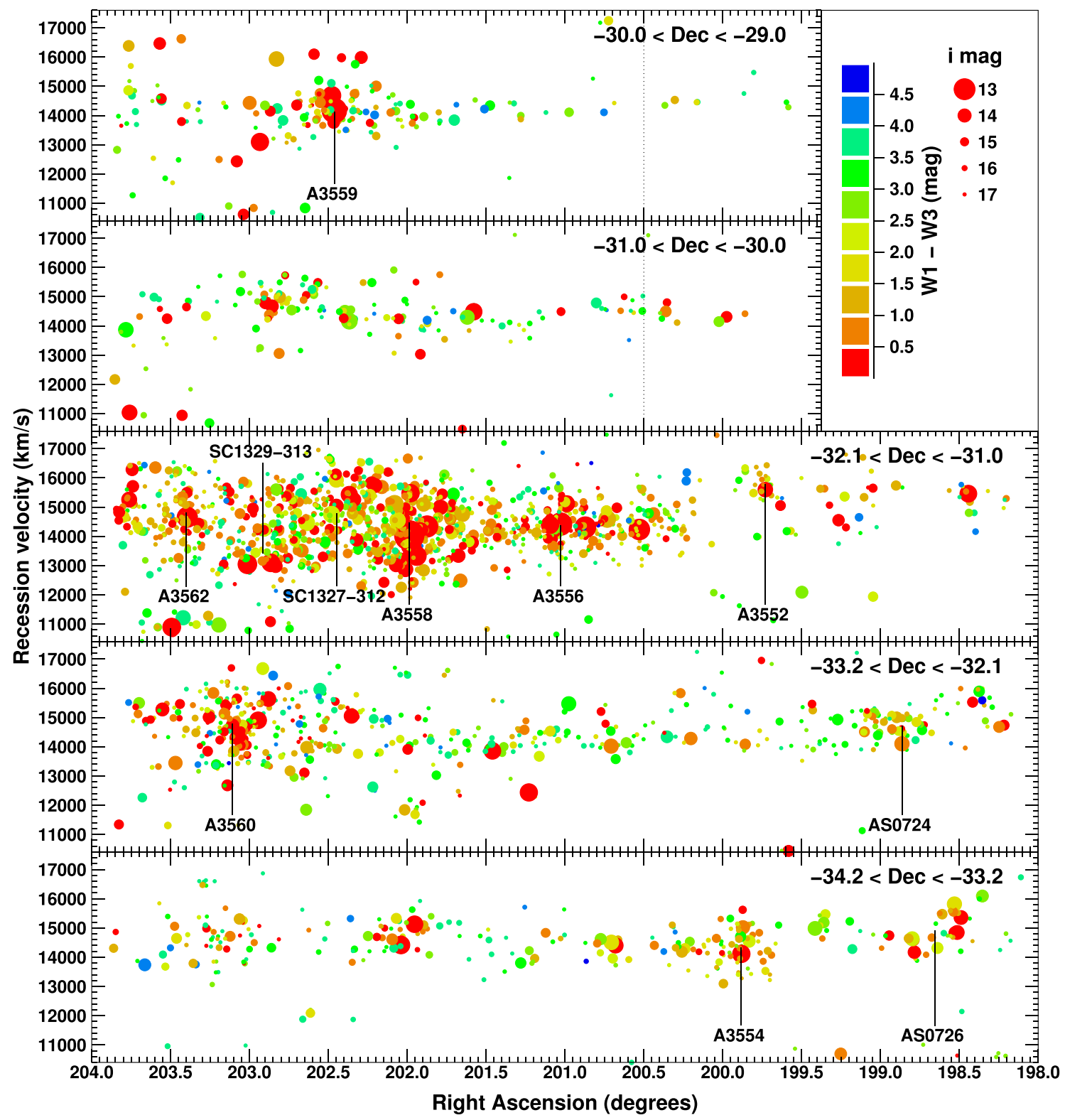

Figure 10. Recession velocity versus right ascension for galaxies across the Shapley supercluster, split into five degree-wide strips in declination. The location and central redshift of each cluster within each declination stripe are labelled. The size and colour of each symbol represent the $i$-band magnitude and $W 1-W 3$ colour of the galaxy as indicated. The dotted lines in the top two stripes mark the RA limit of our AAOmega survey, due to the lack of $i$-band imaging for fields 19 and 20 at the time of the spectroscopic observations. All symbols to the right of these lines are supercluster galaxies with previously known redshifts.

\subsection{A 3D map of the survey volume}

To aid the visualization of the large-scale structures within the surveyed ShaSS volume, we assembled a 3D map associated with all 7170 galaxies in our sample. We adopted the hybrid coordinate system defined in Sutter et al. (2012) to obtain the coordinates $(x$, $y, z)$ of galaxies as follows:

$x=p \times \sin (\phi) \times \cos (\theta)$

$y=p \times \sin (\theta)$

$z=p \times \cos (\phi) \times \cos (\theta)$,

where $p=v_{\mathrm{CMB}} / H_{0}, \phi=\mathrm{RA}-\langle\mathrm{RA}\rangle$ and $\theta=\mathrm{Dec}-\langle\mathrm{Dec}\rangle$. Here $v_{\mathrm{CMB}}=c \times\left(\left(1+z_{\mathrm{CMB}}^{2}\right)-1\right) /\left(\left(1+z_{\mathrm{CMB}}^{2}\right)+1\right)$ and $z_{\mathrm{CMB}}$ is the redshift in the reference frame of the cosmic microwave back- ground. In our adopted orientation, $x$ increases towards the East in the sky, $y$ increases towards the North, and $z$ increases in the radial direction. We further applied a factor $1 / 10$ to the $z$ coordinate for visualization convenience.

We use the X3D pathway (Vogt et al. 2016, 2017) to create an interactive diagram of the resulting 3D map (Fig. 13). This data visualization method relies on the $\mathrm{X} 3 \mathrm{D}$ file format for sharing $3 \mathrm{D}$ models interactively on the World Wide Web, and is natively compatible with all major web browsers without the need for the user to install specific plugins. In the present case, PYTHON and the MAYAVI module (Ramachandran \& Varoquaux 2011) are used to create the $\mathrm{X} 3 \mathrm{D}$ model given the $(x, y, z)$ coordinates of all ShaSS galaxies using the QUIVER3D function. We then create a dedicated interactive experience by wrapping a layer of HTML and JAVASCRIPT around the 


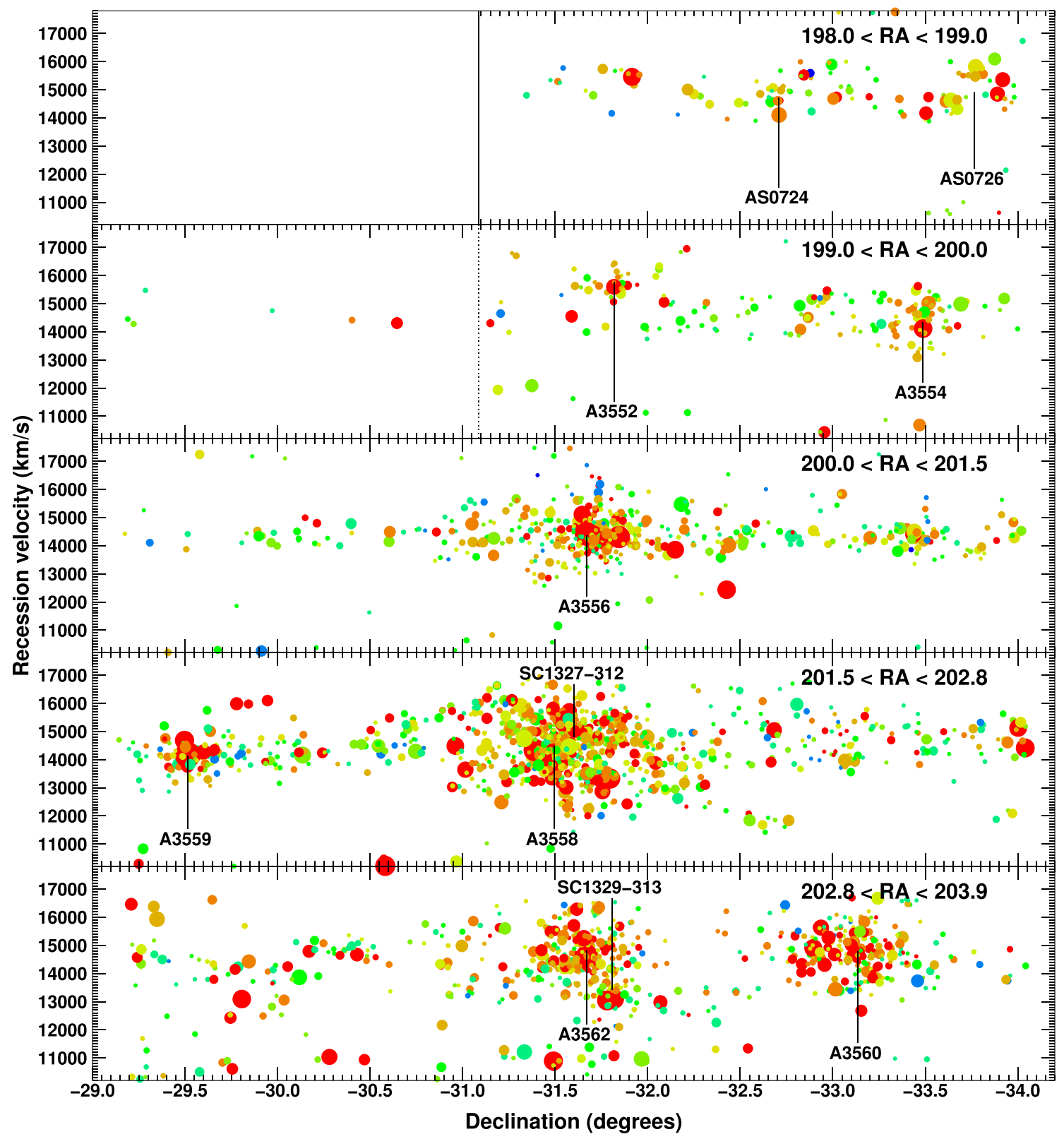

Figure 11. Recession velocity versus declination for galaxies across the SSC, split into five degree-wide strips in right ascension. Symbols and labels as in Fig. 10. The solid line in the top stripe indicates the current limit of the ShaSS data, while the dotted line in the 2nd stripe marks the limit of $i$-band imaging at the time of the AAOmega survey.

X3D model, following the red dice example of Vogt et al. (2016, see Fig. 2).

In the 3D model, each galaxy is represented as a wireframe sphere, colour-coded based on its rest-frame $g-r$ colour. The size of the symbol is proportional to the stellar mass of the galaxy. Coloured wireframe cylinders mark the location of substructures of interest within the Shapley supercluster, and can be toggled on/off using dedicated action buttons. The radius of these cylinders corresponds to the virial radius and the depth corresponds to $3 \times$ the velocity dispersion of the associated substructure. Other action buttons allow the user to toggle the display of galaxies in three rest-frame colour bins $(g-r<0.5,0.5<g-r<0.9,0.9<g-r)$, selected specific viewpoints, from where the model can still be freely manipulated, or take screenshots of the current view.
Finally, we give the user the ability to measure the $z$ coordinate (in Mpc) using clip-planes (Vogt et al. 2017). Two clip-planes can also be used together to display specific slices along the $z$ direction, for example to focus on the Shapley supercluster. The 3D interactive model is available in the online version of this article, and at http: //www.na.astro.it/ShaSS/shapley.html.

\section{CLUSTERS IN THE SHAPLEY SUPERCLUSTER}

We take advantage of the much increased redshift information of Shapley supercluster members to provide updated central redshifts, velocity dispersions, and memberships to each of the 11 clusters within ShaSS that are known to form part of the Shapley superclus- 


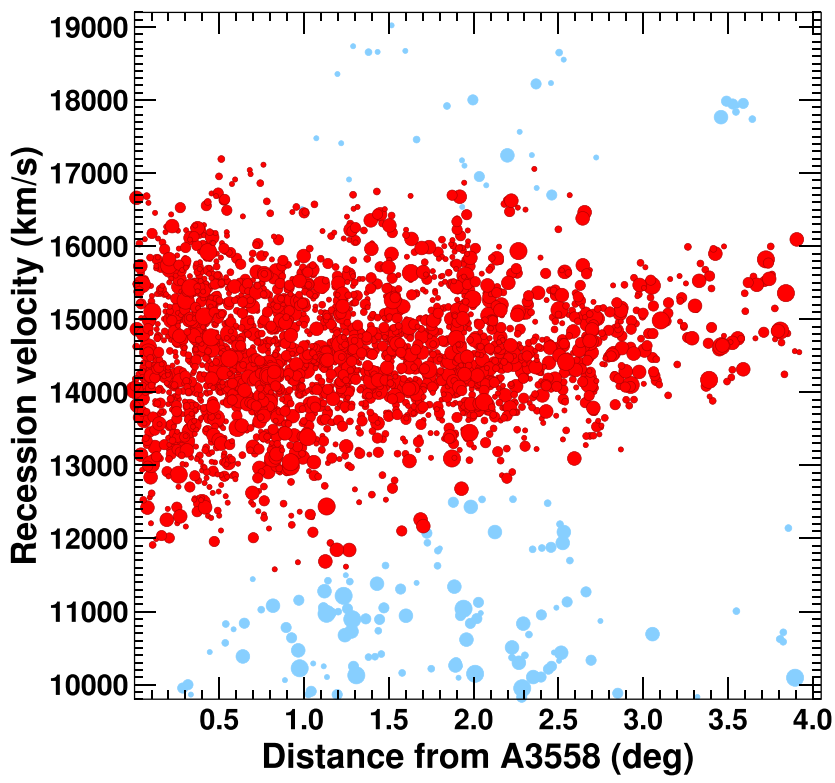

Figure 12. Recession velocity of galaxies versus projected distance from the centre of Abell 3558. The distribution of Shapley supercluster members (red points) shows the typical 'trumpet'-shaped caustic profile, inside which there is a high phase-space density of galaxies. Non-supercluster members are marked in blue.

ter. All 11 of these clusters have been detected at X-ray wavelengths in pointed ROSAT PSPC images of the Shapley supercluster (Ettori et al. 1997; de Filippis et al. 2005) and their X-ray luminosities measured (Table 4). The centres of each cluster (except SC 1329313) are taken to be colocated with the position of the BCG. Taking $1.5 \mathrm{Mpc}$ as a first estimate of $r_{200}$ for the cluster, we use the biweight estimator (Beers, Flynn \& Gebhardt 1990) of the central velocity $\left(C_{\mathrm{BI}}\right)$ and velocity dispersion $\left(S_{\mathrm{BI}}\right)$ of cluster galaxies within $r_{200}$, with an additional $3 \sigma$ clipping to remove obvious outliers. Following Carlberg, Yee \& Ellingson (1997), this velocity dispersion estimate $\left(\sigma_{v}\right)$ and cluster redshift $z_{c l}$ is then used to update the $r_{200}$ estimate as

$r_{200}=2.47 \frac{\sigma_{v}}{1000 \mathrm{~km} \mathrm{~s}^{-1}} \frac{1}{\sqrt{\Omega_{\Lambda}+\Omega_{0}\left(1+z_{c l}\right)^{3}}} h_{70}^{-1} \mathrm{Mpc}$.

The $\sigma_{v}$ and $r_{200}$ values are then iteratively re-estimated until convergence is reached. The virialized regions (inside $r_{200}$ ) and velocity distributions of the interacting clusters A 3562 and SC 1329-313 both overlap, which necessitated additional ad hoc clipping in velocity space to effectively separate the two cluster populations. The locations of these velocity limits were established by examining the range of velocities of galaxies in the cores of each cluster, where the internal velocity distribution of cluster galaxies should be greatest, while minimizing the likelihood of contamination from galaxies in the neighbouring cluster.

Fig. 14 plots the resulting velocity distributions of galaxies within $r_{200}$ of each of the 11 clusters over the full velocity range of supercluster galaxies $\left(11500-17500 \mathrm{~km} \mathrm{~s}^{-1}\right)$. The red histograms indicate the velocity distibutions of those galaxies identified as cluster members, while the yellow histograms mark those galaxies excluded through the clipping procedures. To confirm the reliability of the observed velocity range of cluster galaxies, the dark red histograms show the velocity distributions of those galaxies within $0.5 r_{200}$, i.e. the cluster core. The velocity distributions within $0.5 r_{200}$ and $r_{200}$ can be seen to be consistent.

For comparison the blue curves show the best-fitting Gaussian functions to the velocity distributions, having the same $\sigma_{v}$ and $\left\langle V_{h}\right\rangle$. The velocity distributions for all 11 clusters are shown on the exact same horizontal scale, enabling velocity offsets between clusters to be visualized. The mean velocity of A 3558 (blue dot-dot-dashed line), the central and most massive cluster of the Shapley supercluster, lies within the $1-\sigma$ velocity range of eight of the remaining 10 clusters. The most discrepant velocities are that of A $3552\left(+1277 \mathrm{~km} \mathrm{~s}^{-1}\right.$ relative to A 3558) and SC 1329-313 $\left(-1084 \mathrm{~km} \mathrm{~s}^{-1}\right.$ relative to A 3558$)$. To help substructures to be identified within the velocity distribution of each cluster, the velocities of each galaxy within $r_{200}$ of the cluster are indicated as vertical lines in the barcode-style panels along the top. Significant velocity gaps within the distribution enable the cluster galaxy populations to be partitioned into its component substructures (Beers et al. 1991).

The full details for the 11 clusters are given in Table 4, including position (Cols. 2,3), central velocity $\left\langle V_{h}\right\rangle$ (Col. 4), velocity dispersion (Col. 5), number of spectroscopic members within $r_{200}(\mathrm{Col}$. 6), $r_{200}$ (Col. 7), and X-ray luminosity (Col. 8). The central redshifts and velocity dispersions measured here are fully consistent with those of Moretti et al. (2017) for the three clusters in common: A $3556\left(\left\langle V_{h}\right\rangle=14390 \mathrm{~km} \mathrm{~s}^{-1}, \sigma_{v}=668 \pm 34 \mathrm{~km} \mathrm{~s}^{-1}\right)$, A3358 $(14570,1003 \pm 33)$, and A $3560(14720,840 \pm 35)$.

Ragone et al. (2006) applied a friends-of-friends algorithm to the compilation of redshifts of Proust et al. (2006a) to identify groups of galaxies across the Shapley supercluster, and estimating their masses via their velocity dispersions. They detected 10 of the 11 clusters, and the last three columns of Table 4 present the ID number (Col. 9) in the group catalogue of Ragone et al. (2006), their velocity dispersion estimate (Col. 10) and number of members (Col. 11). They find far fewer cluster members for each system than we do, and in most cases obtain significantly lower $\sigma_{v}$ estimates. They used a shorter linking length than usual to prevent the five clusters in the SSC from merging, and this appears to have had the effect of limiting their memberships to the virialized cores of each cluster, reducing both the numbers and velocity dispersion estimates, particularly in the cases of A 3554, A 3559, AS 0724, and AS 0726. This will likely impact their mass estimates.

Proust et al. (2006a) also estimated the velocity dispersions for each of the 11 clusters, selecting member galaxies as being within $0.5 \mathrm{deg}$ of the X-ray centres. Their $\sigma_{v}$ estimates are in good agreement with ours for seven of the clusters, but obtain much larger velocity dispersion for the clusters A $3562\left(1169 \mathrm{~km} \mathrm{~s}^{-1}\right)$, A $3552\left(682 \mathrm{~km} \mathrm{~s}^{-1}\right)$, SC 1327-312 $\left(857 \mathrm{~km} \mathrm{~s}^{-1}\right)$, and SC 1329$313\left(883 \mathrm{~km} \mathrm{~s}^{-1}\right)$. For the interacting pair of clusters A 3562 and SC 1329-313 their larger estimates are likely due to the difficulty of separating the two cluster populations, while SC 1327-312 remains hard to disentangle from A 3558. These are the four clusters for which we excluded the most galaxies within $r_{200}$ (Fig. 14) when estimating $\left\langle V_{h}\right\rangle$ and $\sigma_{v}$.

While the five clusters in the SSC and Abell 3560 already had excellent redshift coverage, enabling reliable velocity dispersions to be measured and substructures identified, our survey has dramatically improved our knowledge of the other five clusters in the region, doubling their numbers of confirmed spectroscopic members. In the case of Abell 3554, the redshifts for 41 of its 77 members have been measured for the first time by our AAOmega survey, as are 10/19 of the members of Abell 3552, 15/29 members of AS 724 and 18/37 members of AS 726 . 


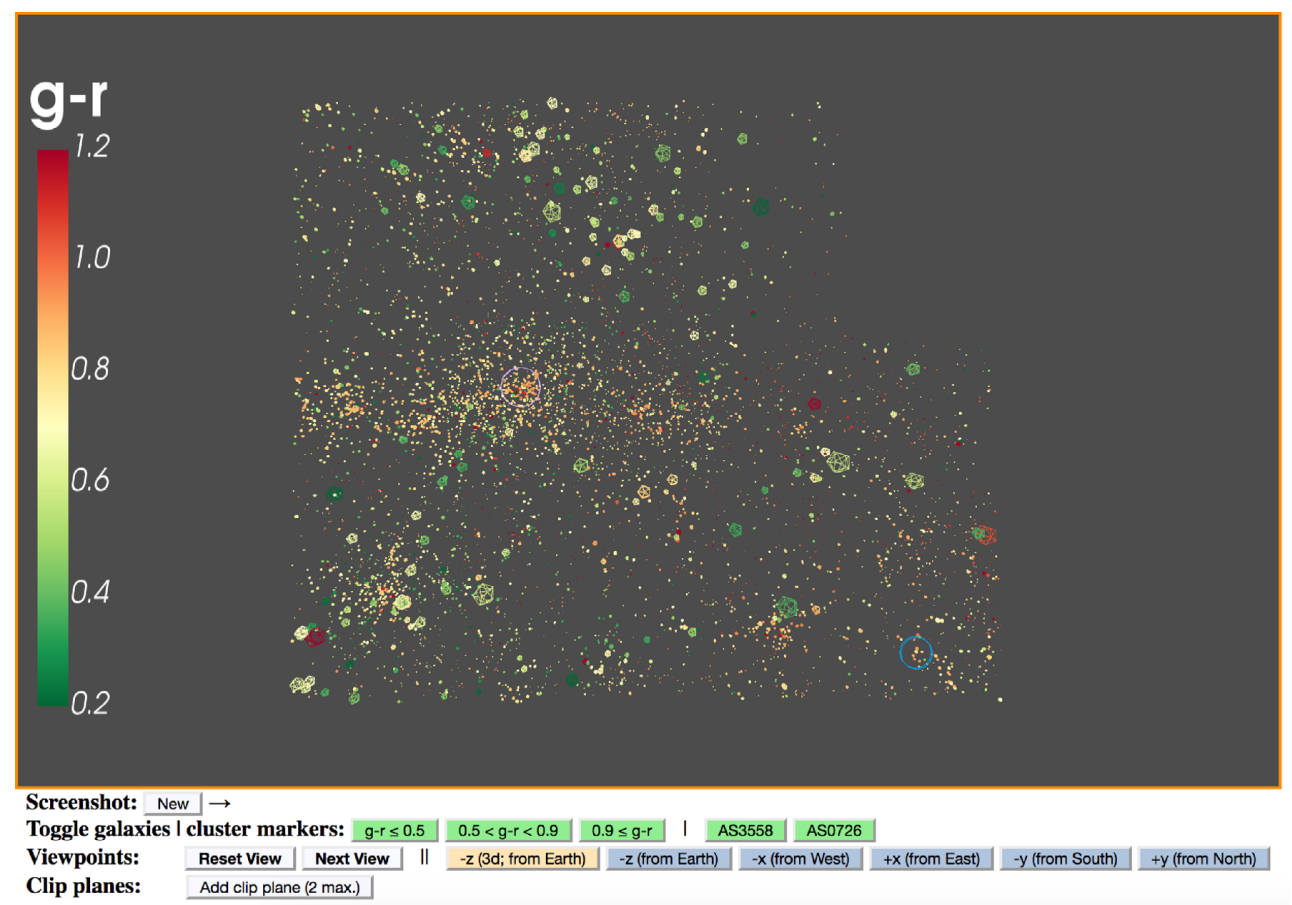

Figure 13. Screenshot of the interactive 3D map of the Shapley Supercluster. Each galaxy is represented by a wireframe sphere, whose colour represents its $g$ $-r$ colour, and size is proportional to its stellar mass. The X3DOM environment enables the user to manually slide, zoom-in, zoom-out and rotate around the model. The interaction buttons shown along the bottom provide additional tools to display/hide galaxy sub-populations based on their $g-r$ colour, display wireframe cylinders representing each of the 11 clusters, move to pre-defined viewpoints, and add clip-planes to display only galaxies within given redshift ranges. An interactive version of this figure is available in the online version of this article, and at http://www.na.astro.it/ShaSS/shapley.html.

Table 4. Properties of the 11 known X-ray galaxy clusters in the Shapley supercluster. The central velocities $\left\langle V_{h}\right\rangle$, velocity dispersions $\sigma_{v}$, number of spectroscopic members within $r_{200}\left(N_{z}\right)$, and $r_{200}$ radii (in Mpc) are all determined using the new extended redshift sample. ROSAT-based X-ray bolometric luminosities taken from de Filippis et al. (2005), except A 3559, A 3560 from Ettori et al. (1997). The last three columns provide the ID number for each cluster in the group catalogue of Ragone et al. (2006), their $\sigma_{v}$ estimates and number of spectroscopic members.

\begin{tabular}{|c|c|c|c|c|c|c|c|c|c|c|}
\hline $\begin{array}{l}\text { Cluster } \\
\text { Name }\end{array}$ & RA (J2000) & Dec (J2000) & $\begin{array}{c}\left\langle V_{h}\right\rangle \\
\left(\mathrm{km} \mathrm{s}^{-1}\right)\end{array}$ & $\begin{array}{c}\sigma_{v} \\
\left(\mathrm{~km} \mathrm{~s}^{-1}\right)\end{array}$ & $N_{z}$ & $\begin{array}{c}r_{200} \\
(\mathrm{Mpc})\end{array}$ & $\begin{array}{c}L_{X, \text { bol }} \\
10^{43} \mathrm{erg} \mathrm{s}^{-1}\end{array}$ & $\begin{array}{c}\text { SSGC } \\
\text { ID }\end{array}$ & $\begin{array}{c}\sigma_{v} \\
\left(\mathrm{~km} \mathrm{~s}^{-1}\right)\end{array}$ & $N_{z}$ \\
\hline Abell 3552 & 199.729560 & -31.817560 & $15777 \pm 75$ & $334 \pm 42$ & 19 & 0.769 & 0.4 & - & - & - \\
\hline Abell 3554 & 199.881979 & -33.488139 & $14346 \pm 66$ & $602 \pm 37$ & 77 & 1.387 & 2.0 & 56 & 323 & 9 \\
\hline Abell 3556 & 201.028020 & -31.669858 & $14396 \pm 45$ & $628 \pm 31$ & 257 & 1.447 & 1.7 & 65 & 491 & 37 \\
\hline Abell 3558 & 201.986896 & -31.495847 & $14500 \pm 39$ & $1007 \pm 25$ & 867 & 2.319 & 66.8 & 73 & 910 & 91 \\
\hline Abell 3559 & 202.462154 & -29.514386 & $14149 \pm 63$ & $521 \pm 39$ & 68 & 1.201 & 1.5 & 84 & 300 & 9 \\
\hline Abell 3560 & 203.107354 & -33.135989 & $14739 \pm 52$ & $860 \pm 32$ & 304 & 1.981 & 11.7 & 88 & 606 & 33 \\
\hline AS 0724 & 198.863788 & -32.710022 & $14748 \pm 74$ & $410 \pm 41$ & 29 & 0.945 & 1.5 & 51 & 350 & 9 \\
\hline SC $1327-312$ & 202.448625 & -31.602450 & $14794 \pm 38$ & $535 \pm 17$ & 220 & 1.347 & 12.7 & 81 & 576 & 26 \\
\hline SC $1329-313$ & 202.913761 & -31.807260 & $13416 \pm 49$ & $373 \pm 28$ & 55 & 0.862 & 5.2 & 83 & 682 & 24 \\
\hline
\end{tabular}

Over the full ShaSS region, 69 per cent of galaxies within the supercluster are members (within $r_{200}$ ) of one of the 11 clusters, while the remaining 31 per cent are identified as intracluster galaxies, located in the wider sheet of galaxies in which the clusters are embedded. This latter value is lower than the 48 per cent found by Proust et al. (2006a) in their analysis of the wider Shapley supercluster region, the difference most likely due to our focus on the densest area of the supercluster, chosen to contain the highest concentration of clusters.

We postpone an analysis estimating updated cluster masses, the overall mass of the Shapley supercluster, and its gravitational pull in the context of the movement of the Local Group relative to the Hubble flow, to a future paper.

\subsection{Comments on individual clusters}

In the following, we discuss some of the properties revealed by our redshift data relating to some of these clusters. 

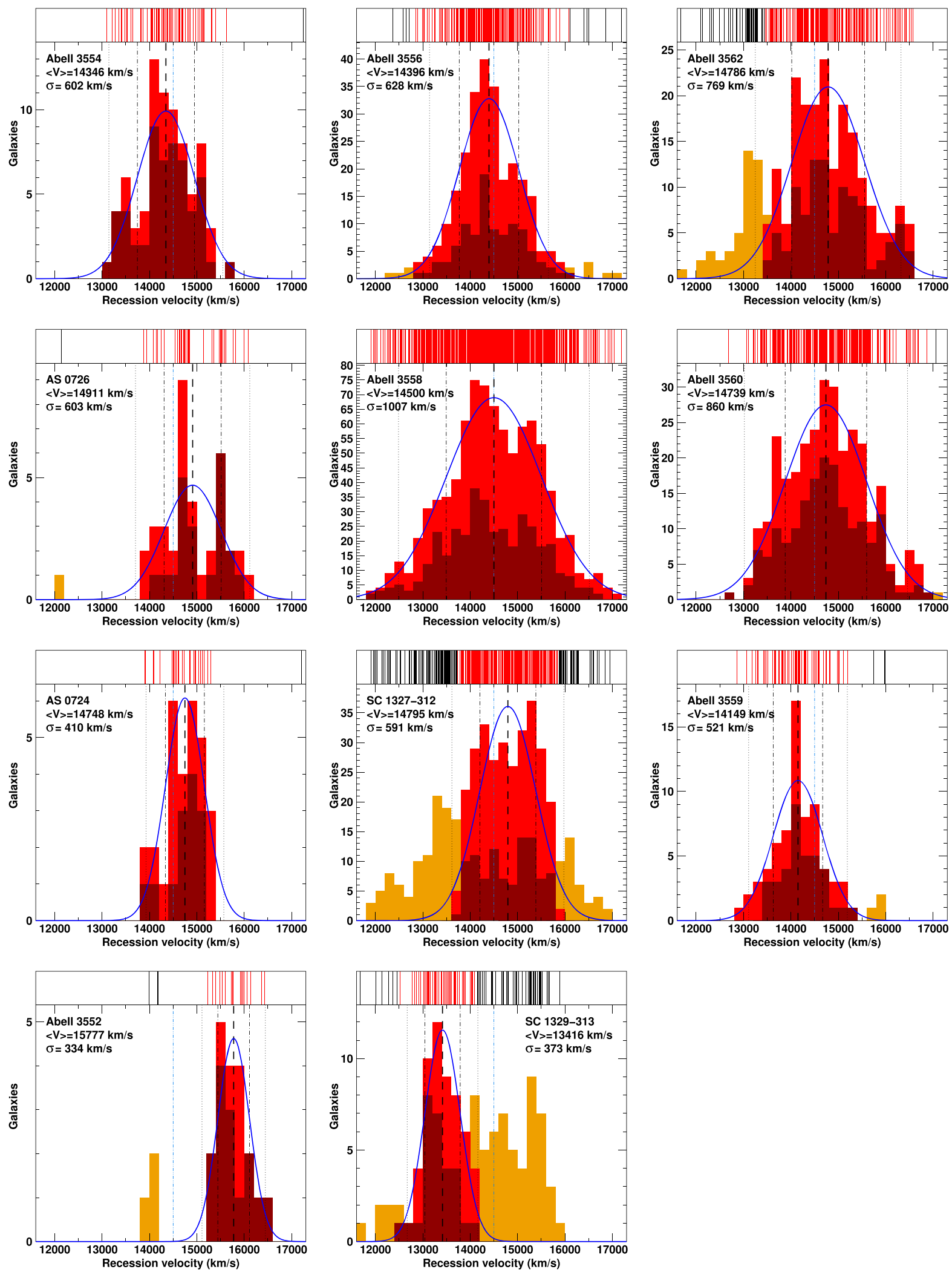

Figure 14. Velocity distributions of galaxies within $r_{200}$ of each of the 11 clusters (red histograms) belonging to the Shapley supercluster. The black dashed line indicates the central recession velocity of each cluster, while the dot-dashed and dotted lines mark the 1- $\sigma$ and 2- $\sigma$ velocity limits. The blue dot-dot-dashed line shows the central velocity of A 3558. The blue curve shows a Gaussian function with the same mean and $\sigma$ as that measured from the cluster galaxies. The dark red histogram shows the velocity distribution of those galaxies within $0.5 r_{2} 00$ of each cluster, and the yellow histogram marks those galaxies within $r_{200}$ that are excluded from the fit. Barcode diagrams along the top of each panel show the velocities of each member (non-member) galaxy within $r_{200}$ of the cluster as red (black) vertical lines. 

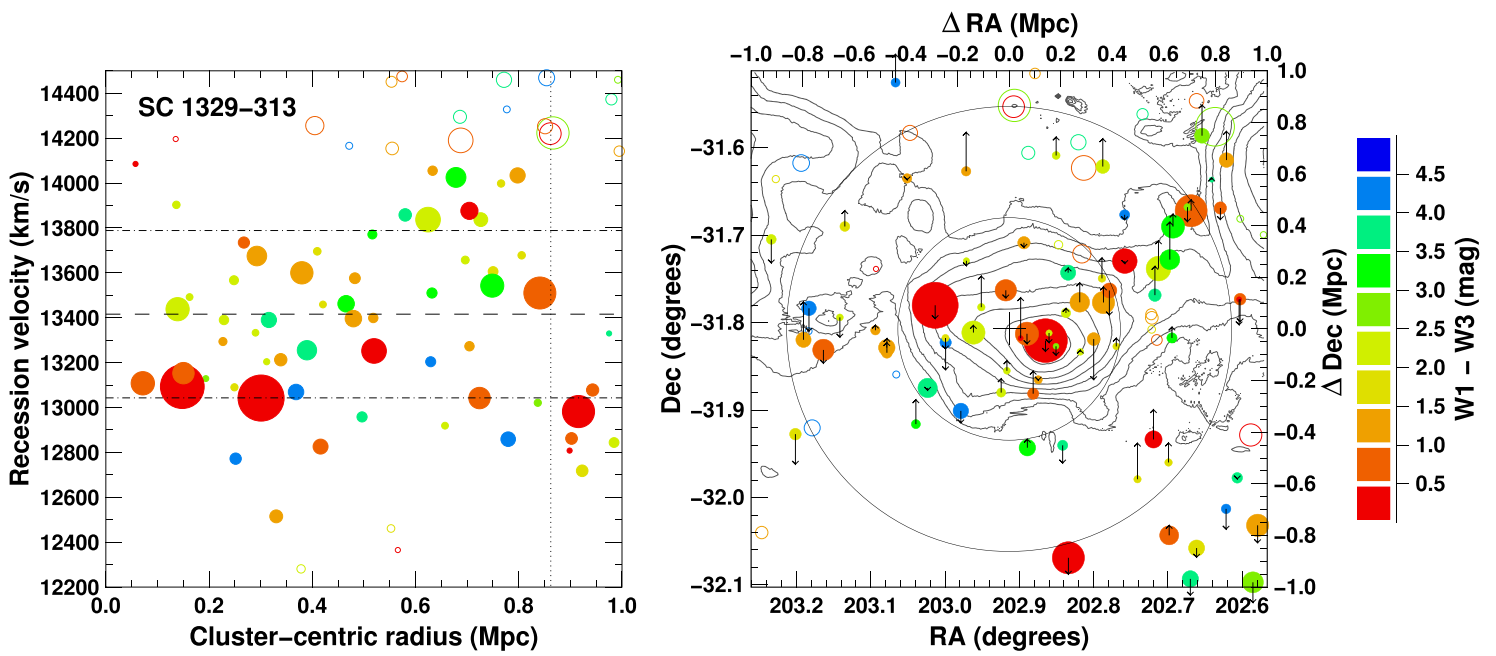

Figure 15. Galaxy cluster SC 1329-313. (Left:) Projected-phase space diagram, $V_{\mathrm{h}}$ versus $r_{\text {proj }}$ for all cluster members (filled symbols) and non-members (open symbols), colour-coded by $W 1-W 3$. The area of the symbols scale with $i$-band luminosity. The dashed and dot-dashed lines indicate the central velocity and $1 \sigma$ velocity dispersion of the members within $r_{200}$ (vertical dotted line). (Right:) Spatial distribution of cluster members (RA, Dec) overlaid on contours of X-ray emission from XMM-Newton. Vertical arrows indicate the LOS velocity relative to the cluster redshift. The large circles indicate $0.5 r_{200}$ and $1.0 r_{200}$.

\subsubsection{SC 1329-313}

SC 1329-313 is an X-ray cluster first detected by the Uhuru satellite and identified in photographic plates by Lugger (1978). A mosaic of XMM-Newton observations showed it to be highly elongated, with a tail extending ENE towards A 3562, and Finoguenov et al. (2004) identified shock fronts, gas sloshing and ridges indicative of a recent high-velocity $\left(1700 \pm 150 \mathrm{~km} \mathrm{~s}^{-1}\right)$ supersonic encounter between A 3562 and SC 1329-313. The peak of the X-ray emission coincides with the bright $(i=13.35)$ early-type galaxy PGC 47548 that has a velocity of $13093 \pm 69 \mathrm{~km} \mathrm{~s}^{-1}$. A similarly bright $(i$ $=13.26$ ) early-type galaxy (PGC 47590) with the same velocity $\left(13042 \pm 81 \mathrm{~km} \mathrm{~s}^{-1}\right)$ is located 8 arcmin to the ENE along the same axis revealed by the $\mathrm{X}$-ray emission, and we take these two galaxies to mark the core of the system, with the centre taken to be $1 / 3$ of the way from PGC 47548 to PGC 47590 . The caustic diagram, $V_{\mathrm{h}}$ versus $r_{\text {proj }}$, (Fig. 15a) reveals numerous other cluster members, whose velocities are mostly slightly above those of the two dominant ellipticals, in the range $12500-14100 \mathrm{~km} \mathrm{~s}^{-1}$. These form a coherent elongated structure in the plane of the sky (Fig. 15b), aligned with the X-ray emission towards A 3562, but changing direction to form a filamentary structure heading NW from the X-ray peak. While there is not an extended velocity gap to higher velocities, those galaxies at $V_{h} \gtrsim 14100 \mathrm{~km} \mathrm{~s}^{-1}$ (open symbols) are not spatially coincident with either the X-ray emission or existing member galaxies, and are instead likely members of A 3562 . We thus consider the velocity limits of SC $1329-313$ to be $12500-14100 \mathrm{~km} \mathrm{~s}^{-1}$, giving a relatively low velocity dispersion of $344 \pm 28 \mathrm{~km} \mathrm{~s}^{-1}$ for the 55 members within $r_{200}$. This approximately matches group T520 from Bardelli et al. (1998a), which has $\left\langle V_{h}\right\rangle=13280 \mathrm{~km} \mathrm{~s}^{-1}$ and $\sigma_{v}=482_{-49}^{+87} \mathrm{~km} \mathrm{~s}^{-1}$.

\subsubsection{AS 0726}

Fig. 14 reveals that the recession velocities of galaxies associated to AS 0726 are far from normally distributed, showing instead two clear sharp peaks at 14700 and $15500 \mathrm{~km} \mathrm{~s}^{-1}$, and a possible third structure at $14200 \mathrm{~km} \mathrm{~s}^{-1}$. The spatial distribution of galaxies in AS 0726 also appears bimodal, with the minor peak at the position given in Proust et al. (2006a) for this system. The barcode plot for AS 0726 shows three significant gaps in velocities, at 14430, 15000 , and $15230 \mathrm{~km} \mathrm{~s}^{-1}$. It seems likely that AS 0726 represents a system of 2-3 groups rather than a single bound entity. Its X-ray emission has however been detected (at 13:15:13.44, - 33:41:42; $L_{X, \text { bol }}=6.9 \times 10^{42} \mathrm{erg} \mathrm{s}^{-1}$; de Filippis et al. 2005), coinciding with the minor peak in the galaxy spatial distribution (Fig. 9) at $14600 \mathrm{~km} \mathrm{~s}^{-1}$. While this appears a relative poor cluster environment, its complex and unrelaxed dynamics could help to explain our recent finding of a massive spiral galaxy within AS 0726, that is undergoing ram-pressure stripping (Merluzzi et al. 2018, in preparation). This process usually requires a high-speed interaction with a dense ICM, but a group-group merger could recreate these conditions by either triggering a shock front in the ICM, or permitting the high encounter velocity between ICM and galaxy.

\subsubsection{Abell 3552}

Abell 3552 appears as a compact, well-defined cluster of 19 galaxies, centred on a dominant elliptical (ShaSS 418015154; 13:18:55.1, $\left.-31: 49: 03, c z=15598 \mathrm{~km} \mathrm{~s}^{-1}\right)$, and X-ray luminosity $L_{X}$,bol $=$ $4.1 \times 10^{42} \mathrm{erg} \mathrm{s}^{-1}$ (de Filippis et al. 2005). Our estimate of the central velocity of the cluster $\left(15777 \mathrm{~km} \mathrm{~s}^{-1}\right)$ is close to that originally estimated by Quintana et al. (2000), but significantly higher than the value of $14753 \pm 119 \mathrm{~km} \mathrm{~s}^{-1}$ given by Proust et al. (2006a). This latter estimate appears incorrect, since we find no galaxies with velocities in the range $14250-15200 \mathrm{~km} \mathrm{~s}^{-1}$. Our velocity dispersion estimate $\left(334 \pm 42 \mathrm{~km} \mathrm{~s}^{-1}\right)$ is also much lower than the $682 \mathrm{~km} \mathrm{~s}^{-1}$ value of Proust et al. (2006a). The cluster is embedded in a filament orientated E-W, connecting the core of the supercluster with the A $3528 /$ A 3532 complex $\sim 6^{\circ}$ to the West.

\subsubsection{Abell 3559}

Abell $3559\left(L_{X, \text { bol }}=1.5 \times 10^{43} \mathrm{erg} \mathrm{s}^{-1}\right.$; Ettori et al. 1997) is centred on a complex system of five early-type galaxies including a dominant central Elliptical (ShaSS 410043635; 13:29:50.9, -29:30:52; $c z=14168 \mathrm{~km} \mathrm{~s}^{-1}$ ). Fig. 9 reveals a $\mathrm{N}-\mathrm{S}$ filamentary structure (RA 13:31:00) connecting A 3559 with the Shapley supercluster 


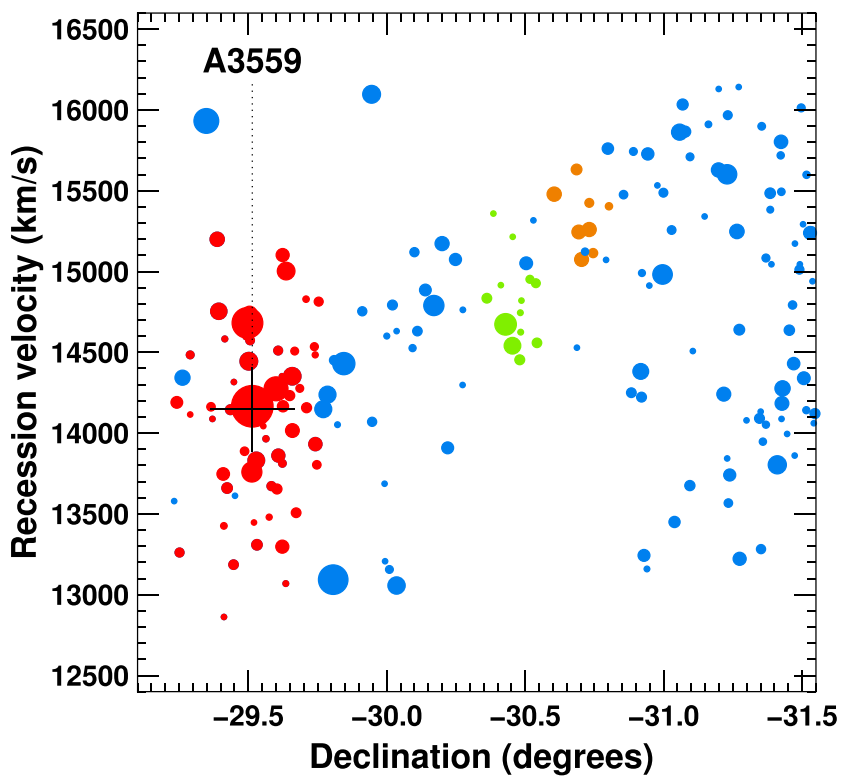

Figure 16. Velocity structure of galaxies in the filament connecting A 3559 and the SSC. Galaxies within the N-S filament $\left(202.5^{\circ}<R A<203.1^{\circ}\right)$ are shown as blue symbols. Red points mark A 3559 cluster members. The black cross marks the central velocity and Declination of the cluster. Green and orange points mark members of the two groups embedded in the filament.

core. Fig. 16 shows the velocity structure of galaxies along this filament. The member galaxies of A 3559 (red points) are seen to scatter about a mean velocity of $14169 \mathrm{~km} \mathrm{~s}^{-1}$. Moving South from the cluster, a filament of galaxies (blue points) starts at $\sim 14000 \mathrm{~km} \mathrm{~s}^{-1}$ and shows a steady velocity gradient, reaching $\sim 16000 \mathrm{~km} \mathrm{~s}^{-1}$ at Dec -31.3 where it meets the SSC. Two galaxy groups are embedded within this connecting filament. A first group centred on a bright early-type galaxy (ShaSS 404047322; 13:31:25.7, -30:25:45; $c z=$ $14675 \mathrm{~km} \mathrm{~s}^{-1}$; Filament Group A in Fig. 9) and containing 13 members (green points; $\left\langle V_{h}\right\rangle=14805 \mathrm{~km} \mathrm{~s}^{-1} ; \sigma=249 \mathrm{~km} \mathrm{~s}^{-1}$ ), and a second system of eight galaxies at 13:30:22, $-30: 42: 40$ (orange points; $\left\langle V_{h}\right\rangle=15327 \mathrm{~km} \mathrm{~s}^{-1}, \sigma=188 \mathrm{~km} \mathrm{~s}^{-1}$; Filament Group B in Fig. 9). Such a velocity gradient is consistent with A 3559 being on the near side of the SSC, and galaxies in the filament being accelerated as they approach the SSC, reaching infall velocities of $\sim 2000 \mathrm{~km} \mathrm{~s}^{-1}$ (cf. Falco et al. 2014; Lee, Kim \& Rey 2015; Kim et al. 2016).

\subsubsection{Abell 3560}

Fig. 9 reveals clear substructure in Abell 3560, with secondary peaks 18 arcmin to the North and 25 arcmin to the West of the cluster centre, which we refer to as A 3560-N and A 3560-W, respectively. The analysis of XMM X-ray imaging of A 3560 by Venturi et al. (2013) indicated it to be undergoing a minor merger, with asymmetric emission, a positional shift between the inner and outer isophotes, a spiral-like structure suggestive of gas sloshing, and a sub-group $\sim 8^{\prime}$ NW from the cluster centre, mid-way towards our group A $3560-\mathrm{N}$

A 3560-N appears centred on a close pair of early-type galaxies (13:32:14.1, -32:49:56). A 3560-W is centred on a bright elliptical (ShaSS 406059814; 13:30:32.8, -33:03:52, $c z=13976 \mathrm{~km} \mathrm{~s}^{-1}$ ). The group consists of 20 galaxies with a velocity dispersion of $286 \mathrm{~km} \mathrm{~s}^{-1}$.

\subsubsection{Abell 3556}

The galaxy density map of Fig. 9 reveals a new group of galaxies approximately midway between A 3556 and A 3552 (labelled as A3556-W). This group is centred on a dominant elliptical galaxy (ShaSS 413015757; 13:22:06.7, -31:46:16; $c z=14234 \mathrm{~km} \mathrm{~s}^{-1}$ ). The group consists of 28 galaxies with a velocity dispersion of $247 \mathrm{~km} \mathrm{~s}^{-1}$. It was not covered (too far West) by the substructure analysis of A 3556 by Bardelli et al. (1998b), or identified as a group by Ragone et al. (2006).

\section{THE REDSHIFT CATALOGUE}

In total, we have obtained reliable redshifts $(Q \geq 3)$ for 4027 unique galaxies through our AAOmega observations. The complete catalogue of these redshifts is published here in Table 5 and will be available through the CDS. The first column provides the unique identification key (SHASS_ID) inherited from the $i$-band photometric catalogue of Mercurio et al. (2015), enabling all the photometric properties of each galaxy to be univocally obtained from the ShaSS database. This service is publicly available via browser at the address http://shass.na.astro.it. The next three columns give the sky coordinates and $i$-band Kron magnitude of each galaxy as obtained from our $i$-band photometry. The last two columns give the redshift and its uncertainty measured from the AAOmega spectra.

\section{SUMMARY}

We have mapped the structure of the Shapley supercluster and its surroundings as traced by the distribution of its member galaxies. To achieve this, we have completed a new redshift survey of galaxies in a 21 square degree region of the Shapley Supercluster based on observations carried out with the AAOmega spectrograph on the 3.9$\mathrm{m}$ Anglo-Australian Telescope. This is the spectroscopic component of the Shapley Supercluster Survey (Merluzzi et al. 2015), which has acquired high-quality optical ugri and near-infrared $K$-band imaging with the 2.6-m VST and 4.1-m VISTA telescopes.

We have targetted galaxies from the VST images with $i<18.0$ and $W 1<15.5 \mathrm{mag}$, and present a final redshift sample of 4027 galaxies with reliable redshifts. The high quality of the spectra acquired has meant that we could obtain reliable redshifts for 98.1 per cent of the targets observed, with mean internal errors of $51 \mathrm{~km} \mathrm{~s}^{-1}$ for each

Table 5. Galaxy redshifts. The first rows of this table are presented here for guidance regarding its form and content.

\begin{tabular}{lccccc}
\hline ShaSS_ID & $\begin{array}{c}\text { RA (J2000) } \\
(\text { degrees })\end{array}$ & $\begin{array}{c}\text { Dec (J2000) } \\
\text { (degrees) }\end{array}$ & $\begin{array}{c}i \text {-band } \\
(\mathrm{mag})\end{array}$ & $z$ & $z_{\text {err }}$ \\
\hline 421013127 & 198.1498561 & -33.8295585 & 16.759 & 0.13266 & 0.00017 \\
421039180 & 198.1536289 & -33.3997827 & 16.776 & 0.07809 & 0.00019 \\
422024665 & 198.1548889 & -32.8054486 & 17.341 & 0.21635 & 0.00088 \\
422046830 & 198.1550620 & -32.5114358 & 15.547 & 0.13089 & 0.00021 \\
\hline
\end{tabular}


individual measurement, and a blunder rate of just 0.2 per cent. After merging these new redshifts with those from the literature, 95 per cent of galaxies with $i<18.0$ and $W 1<15.5$ mag over the $21 \mathrm{deg}^{2}$ region have reliable redshifts.

This redshift sample has enabled us to determine updated mean redshifts, memberships and velocity dispersions for the 11 known X-ray clusters from the Shapley supercluster covered by ShaSS. The much improved homogeneity and completeness of the redshift coverage has enabled the connecting filamentary structures to be mapped in much greater detail and depth. All 11 clusters are shown to be inter-connected by these filaments, and lie embedded within a coherent sheet of galaxies at $14500 \mathrm{~km} \mathrm{~s}^{-1}$ that fills the entire survey region without gaps. The well-defined caustics extending to the survey limits, lack of significant velocity gradients within the sheet, along with the clear voids either side of this sheet, all confirm that the entire region has detached itself from the wider Hubble expansion, is gravitationally bound and is in the process of steady collapse to form a single virialized system in the distant future.

\section{ACKNOWLEDGEMENTS}

CPH acknowledges financial support from PRIN INAF 2014 and CONICYT Anillo project ACT-1122. This work is based on data collected with the Anglo-Australian Telescope and 2dF+AAOmega at the Australian Astronomical Observatory, Australia. These observations have been financed by the OPTICON consortium (proposal 2013A/014). The authors thank Dr. A. M. Hopkins for the assistance during the observation run and Dr. A. Moretti and the OmegaWINGS team to share their redshift measurements in advance of publication. The optical imaging is collected at the VLT Survey Telescope using the Italian INAF Guaranteed Time Observations (ESO programmes 088.A-4008, 089.A-0095, 090.A-0094). PM, GB, and AM acknowledge financial support from PRIN-INAF2014: Galaxy Evolution from Cluster Cores to Filaments (PI B.M. Poggianti) and INAF PRIN-SKA 2017 ESKAPE (PI L. Hunt). KAP acknowledges support of STFC through the University of Hull Consolidated Grant ST/R000840/1. MAD acknowledges the support of the Australian Research Council (ARC) through Discovery project DP16010363. This publication makes use of data products from WISE, which is a joint project of the University of California, Los Angeles, and the Jet Propulsion Laboratory/California Institute of Technology, funded by the National Aeronautics and Space Administration.

\section{REFERENCES}

Araya-Melo P. A., Reisenegger A., Meza A., van de Weygaert R., Dünner R., Quintana H., 2009, MNRAS, 399, 97

Bahé Y. M., McCarthy I. G., Balogh M. L., Font A. S., 2013, MNRAS, 430, 3017

Baldry I. K. et al., 2018, MNRAS, 474, 3875

Bardelli S., Zucca E., Vettolani G., Zamorani G., Scaramella R., Collins C. A., MacGillivray H. T., 1994, MNRAS, 267, 665

Bardelli S., Pisani A., Ramella M., Zucca E., Zamorani G., 1998a, MNRAS, 300,589

Bardelli S., Zucca E., Zamorani G., Vettolani G., Scaramella R., 1998b, MNRAS, 296, 599

Bardelli S., Zucca E., Zamorani G., Moscardini L., Scaramella R., 2000, MNRAS, 312, 540

Beers T. C., Flynn K., Gebhardt K., 1990, AJ, 100, 32

Beers T. C., Gebhardt K., Forman W., Huchra J. P., Jones C., 1991, AJ, 102, 1581

Bianconi M., Smith G. P., Haines C. P., McGee S. L., Finoguenov A., Egami E., 2018, MNRAS, 473, L79
Bond J. R., Kofman L., Pogosyan D., 1996, Nature, 380, 603

Cannon R. et al., 2006, MNRAS, 372, 425

Capaccioli M., Schipani P., 2011, ESO Messenger, 146, 2

Carlberg R. G., Yee H. K. C., Ellingson E., 1997, ApJ, 478, 462

Cava A. et al., 2009, A\&A, 495, 707

Chow-Martínez M., Andernach H., Caretta C. A., Trejo-Alonso J. J., 2014, MNRAS, 445, 4073

Cluver M. E., Jarrett T. H., Dale D. A., Smith J.-D. T, August T., Brown M. J. I., 2017, ApJ, 850, 68

Colless M. et al., 2001, MNRAS, 328, 1039

Courtois H. M., Tully R. B., Hoffman Y., Pomarède D., Graziani R., Dupuy A., 2017, ApJL, 847, L6

de Filippis E., Schindler S., Erben T., 2005, A\&A, 444, 387

Diaferio A., 1999, MNRAS, 309, 610

Diaferio A., Geller M. J., 1997, ApJ, 481, 633

Drinkwater M. J., Proust D., Parker Q., Quintana H., Slezak E., 1999, PASA, 16,113

Drinkwater M. J., Parker Q., Proust D., Slezak E., Quintana H., 2004, PASA, 21,89

Drinkwater M. J. et al., 2010, MNRAS, 401, 1429

Driver S. P. et al., 2011, MNRAS, 413, 971

Dünner R., Araya P. A., Meza A., Reisenegger A., 2006, MNRAS, 366, 803

Einasto M., Einasto J., Tago E., Müller V., Andernach H., 2001, AJ, 122, 2222

Einasto M., Liivamägi L. J., Tago E., Saar E., Tempel E., Einasto J., Martínez V. J., Heinämäki P., 2011a, A\&A, 532, 5

Einasto M. et al., 2011b, ApJ, 736, 51

Einasto M. et al., 2016, A\&A, 595, 70

Ettori S., Fabian A. C., White D. A., 1997, MNRAS, 289, 787

Falco M., Hansen S. H., Wojtak R., Brinckmann T., Lindholmer M., Pandolfi S., 2014, MNRAS, 442, 1887

Finoguenov A., Henriksen M. J., Briel U. G., de Plaa J., Kaastra J. S., 2004, ApJ, 611, 811

Gargiulo A. et al., 2009, MNRAS, 397, 75

Gott J. R., III, Melott A. L., Dickinson M., 1986, ApJ, 306, 341

Grado A., Capaccioli M., Limatola L., Getman F., 2012, Mem. SAIt, 19, 362

Guzzo L. et al., 2014, A\&A, 566, A108

Haines C. P., Merluzzi P., Mercurio A., Gargiulo A., Krusanova N., Busarello G., La Barbera F., Capaccioli M., 2006, MNRAS, 371, 55

Haines C. P., Gargiulo A., La Barbera F., Mercurio A., Merluzzi P., Busarello G., 2007, MNRAS, 381, 7

Haines C. P., Gargiulo A., Merluzzi P., 2008, MNRAS, 385, 1201

Haines C. P., Busarello G., Merluzzi P., Smith R. J., Raychaudhury S., Mercurio A., Smith G. P., 2011a, MNRAS, 412, 127

Haines C. P., Busarello G., Merluzzi P., Smith R. J., Raychaudhury S., Mercurio A., Smith G. P., 2011b, MNRAS, 412, 145

Haines C. P., Busarello G., Merluzzi P., Smith R. J., Raychaudhury S., Mercurio A., Smith G. P., 2011c, MNRAS, 417, 145

Haines C. P. et al., 2013, ApJ, 775, 126

Haines C. P. et al., 2015, ApJ, 806, 101

Haines C. P. et al., 2018, MNRAS, 477, 4931

Huchra J. P., Macri L. M. et al., 2012, ApJS, 199, 26

Jaffé Y. L. et al., 2016, MNRAS, 461, 1202

Jarrett T. H., Chester T., Cutri R., Schneider S., Skrutskie M., Huchra J. P., 2000, AJ, 119, 2498

Joeveer M., Einasto J., 1978, in Longair M. S., Einasto J., eds, Proc. IAU Symp. 79, Large Scale Structures in the Universe. Kluwer, Dordrecht, p. 241

Jones D. H., et al., 2009, MNRAS, 399, 683

Kaldare R., Colless M., Raychaudhury S., Peterson B. A., 2003, MNRAS, 339,652

Kim S. et al., 2016, ApJ, 833, 207

Kleiner D., Pimbblet K. A., Jones D. H., Koribalski B. S., Serra P., 2017, MNRAS, 466, 4692

Kocevski D. D., Mullis C. R., Ebeling H., 2004, ApJ, 608, 721

Kuijken K., 2011, ESO Messenger, 146, 8

Lee J., Kim S., Rey S.-C., 2015, ApJ, 807, 122 
Leroy A. K., Walter F., Brinks E., Bigiel F., de Blok W. J. G., Madore B., Thornley M. D., 2008, AJ, 136, 2782

Lewis I. J. et al., 2002, MNRAS, 333, 279

Liske J. et al., 2015, MNRAS, 452, 2087

Lugger P. M., 1978, ApJ, 221, 745

Mahajan S., Haines C. P., Raychaudhury S., 2010, MNRAS, 404, 1745

Mamon G. A., Sanchis T., Salvador-Solé E., Solanes J. M., 2004, A\&A, 414,445

McGaugh S. S., Schombert J. M., 2014, AJ, 148, 77

Mercurio A. et al., 2006, MNRAS, 368, 109

Mercurio A. et al., 2015, MNRAS, 453, 3685

Merluzzi P., Mercurio A., Haines C. P., Smith R. J., Busarello G., Lucey J. R., 2010, MNRAS, 402, 753

Merluzzi P. et al., 2013, MNRAS, 429, 1747

Merluzzi P. et al., 2015, MNRAS, 446, 803

Merluzzi P., Busarello G., Dopita M. A., Haines C. P., Steinhauser D., Bourdin H., Mazzotta P., 2016, MNRAS, 460, 3345

Merluzzi P., Busarello G., Dopita M. A., Thomas A. D., Haines C. P., Grado A., Limatola L., Mercurio A., 2018, ApJ, 852, 113

Moretti A. et al., 2017, A\&A, 599, A81

Norberg P. et al., 2002, MNRAS, 336, 907

Pearson D. W., 2015, MNRAS, 449, 3212

Pimbblet K. A., 2011, MNRAS, 411, 2637

Pimbblet K. A., Drinkwater M. J., Hawkrigg M. C., 2004, MNRAS, 354, L61

Pisani A., 1993, MNRAS, 265, 706

Pisani A., 1996, MNRAS, 278, 697

Planck Collaboration, 2014, A\&A, 571, 29 Planck 2013 results. XXIX

Porter S. C., Raychaudhury S., Pimbblet K. A., Drinkwater M. J., 2008, MNRAS, 388, 1152

Proust D. et al., 2006a, A\&A, 447, 133

Proust D. et al., 2006b, ESO Messenger, 124, 30

Quintana H., Ramirez A., Melnick J., Raychaudhury S., Slezak E., 1995, AJ, 110, 463

Quintana H., Melnick J., Proust D., Infante L., 1997, A\&ASS, 125, 247

Quintana H., Carrasco E. R., Reisenegger A., 2000, AJ, 120, 511

Radburn-Smith D. J., Lucey J. R., Woudt P. A., Kraan-Korteweg R. C., Watson F. G., 2006, MNRAS, 369, 1131

Ragone C. J., Muriel H., Proust D., Reisenegger A., Quintana H., 2006, A\&A, 445, 819
Ramachandran P., Varoquaux G., 2011, IEEE Comput. Sci. Eng., 13, 40

Raychaudhury S., 1989, Nature, 342, 251

Regös E., Geller M. J., 1989, AJ, 98, 755

Reisenegger A., Quintana H., Carrasco E. R., Maze J., 2000, AJ, 120, 523

Rossetti M., Ghizzardi S., Molendi S., Finoguenov A., 2007, A\&A, 463, 839

Shapley H., 1930, Harv. Coll. Obs. Bull., 874, 9

Sharp R. et al., 2006, Proc. SPIE, 6269, 62690G

Silverman B. W., 1986, Density Estimation for Statistics and Data Analysis. Chapman and Hall, London

Smith G. A. et al., 2004a, Proc. SPIE, 5492, 410

Smith R. J. et al., 2004b, AJ, 128, 1558

Smith R. J., Lucey J. R., Hudson M. J., 2007, MNRAS, 381, 1035

Springel V., Frenk C. S., White S. D. M., 2006, Nature, 440, 1137

Sutter P. M., Lavaux G., Wandelt B. D., Weinberg D. H., 2012, ApJ, 761, 44

Venturi T. et al., 2013, A\&A, 558, 146

Vogt F. P. A., Owen C. I., Verdes-Montenegro L., Borthakur S., 2016, ApJ, 818,115

Vogt F. P. A., Seitenzahl I. R., Dopita M. A., Ruiter A. J., 2017, PASP, 129, 8012

Wolf C. et al., 2009, MNRAS, 393, 1302

Wright E. L. et al., 2010, AJ, 140, 1868

Zeldovich I. B., Einasto J., Shandarin S. F., 1982, 300, 407

\section{SUPPORTING INFORMATION}

Supplementary data are available at MNRAS online.

Table 5. The full redshift catalogue from the Shapley Supercluster Survey. (1) ShaSS ID number of galaxy, (2,3) Right Ascension and Declination (J2000) in degrees, (4) i-band magnitude (AB), (5, 6)

redshift, z, and uncertainty.

Please note: Oxford University Press is not responsible for the content or functionality of any supporting materials supplied by the authors. Any queries (other than missing material) should be directed to the corresponding author for the article.

This paper has been typeset from a $\mathrm{T}_{\mathrm{E}} \mathrm{X} / \mathrm{L} \mathrm{T}_{\mathrm{E}} \mathrm{X}$ file prepared by the author. 